\title{
Efficient and Incentive Compatible Exchange of Real-Time Information
}

\author{
William Phan \\ University of Helsinki and HECER
}

Discussion Paper No. 410

March 2017

ISSN 1795-0562

HECER - Helsinki Center of Economic Research, P.O. Box 17 (Arkadiankatu 7), FI-00014 University of Helsinki, FINLAND,

Tel +358-2941-28780, E-mail info-hecer@helsinki.fi, Internet www.hecer.fi 


\title{
Efficient and Incentive Compatible Exchange of Real-Time Information*
}

\begin{abstract}
We consider the problem of coordinating the exchange of real-time information. For example, a US Department of Transportation pilot program seeks to reduce traffic accidents by allowing each vehicle to transfer crash-relevant information (e.g. position, speed, braking status) to and from neighboring vehicles. Time is of the essence: vehicle information becomes stale quickly. Electronic files are the medium of information and corrupt/useless when partial; hence, we model them as discrete, indivisible objects. Furthermore, each agent may perfectly replicate an object in his possession and transfer it to another agent. However, replication and transfer takes time (e.g. due to bandwidth constraints), and scarcity arises due to fact that information quickly becomes valueless. How should agents transfer such objects? We study efficiency, strategy-proofness, withholding-proofness, and introduce a new axiom based on the concept of reciprocity. Our results are as follows:

When each agent owns one object and consumes only one object, we identify a family of rules satisfying all four axioms. If each agent owns a bundle of objects and consumes a bundle, then the four axioms are incompatible. If agents live in a network, then for a large class of incomplete networks, efficiency is only compatible with strategy-proofness.
\end{abstract}

JEL Classification: D47, D63, D70

Keywords: replication, electronic file, object, strategy-proofness, reciprocity

William Phan

Department of Political and Economic Studies

University of Helsinki

P.O. Box 17 (Arkadiankatu 7)

FI-00014 University of Helsinki

FINLAND

e-mail: william.phan@helsinki.fi

* I thank Srihari Govindan, Ryan Tierney, Heng Liu, participants at the 2013 Conference on Economic Design, the 2013 Osaka Rochester Theory Workshop, and the 24th International Workshop on Game Theory. I am especially grateful for comments from Vikram Manjunath, Takayuki Oishi, and William Thomson. 


\title{
Efficient and Incentive Compatible Exchange of Real-Time Information
}

\author{
William Phan*
}

March 14, 2017

\section{Introduction}

An agent in possession of an electronic file may produce a perfect copy and transfer this copy to another agent. This is not a standard notion of production in economics. We propose and study a resource allocation problem where agents exchange replicable objects, and time is of the essence. We consider two forms of incentive compatibility-agents always 1) report their true preference, and 2) fully bring to the table the resources they have. Additionally, we propose a fairness criterion based on the concept of reciprocity. That is, we reward an agent for contributing to others' welfare in a reciprocal manner; however, the way in which he contributes is determined endogenously by the rule. When objects are not replicable, Gale's Top Trading Cycles rule satisfies several important criteria. We extend this positive finding by showing that in our new environment there is a family of rules satisfying all of the aforementioned properties.

Our example is motivated by the following statistic:

"Motor vehicle travel is the primary means of transportation in the United States, providing an unprecedented degree of mobility. Yet for all its advantages, motor vehicle crashes are the leading cause of death for age 4 and every age 11 through 27 (based on 2009 data). The mission of the National Highway Traffic Safety Administration is to reduce deaths, injuries, and economic losses from motor vehicle crashes."

-National Highway Traffic Safety Administration ${ }^{1}$

The Vehicle Safety Communications-Applications program, run by the U.S. Department of Transportation's National Highway Traffic Safety Administration and supported by a consortium of major car manufacturers, has developed and tested a system whereby each vehicle broadcasts basic information (e.g. position, speed, acceleration, etc.) to surrounding vehicles (Ahmed-Zaid et al., 2011). Such information is used as a crash counter-measure, alerting drivers to engage in some sort of safety action. For example, a driver begins a

${ }^{*}$ Department of Political and Economic Studies, University of Helsinki. E-mail: william.phan@helsinki.fi.

${ }^{1}$ Traffic Safety Facts, NHTSA (April 2014). DOT HS 812016. 
maneuver to pass a slower vehicle in front, a vehicle approaching from the opposite direction broadcasts its presence, and the driver's dashboard flashes a warning preventing a possible collision. One disadvantage, however, is that each vehicle must listen to all broadcasts; so messages are kept short to prevent congestion. ${ }^{2}$

We consider the case of a second layer of information exchange where vehicles may request and send longer messages (e.g. path history, path prediction) in a targeted fashion as opposed to indiscriminate broadcasts. ${ }^{3}$ Targeted messages are not a technical constraint, but a conscious choice to prevent congestion. ${ }^{4}$ For example, upon learning from the primary broadcast layer that Vehicle B is approaching from the other direction, Vehicle A can request more detailed information (e.g. Vehicle B diagnostics, path projection, path history (potholes, patches of ice, debris), radar/sensor-gathered information). In this sense, Vehicle A's request in the second layer is some function of his planned route and the information gleaned in the primary broadcast layer. A central computer (possibly a road-side unit) collects the requests, and recommends a process of exchange between agents. Our paper studies such recommendations from an economic perspective. ${ }^{5}$

Scarcity arises from the tension between two properties of real-time information: the ability to be replicated versus decaying value. Information is sent via an electronic message in a wireless network (much like routers and laptops); capacity constraints of wireless communication limit the rate of information sent. By virtue of the replicability of electronic data, though, a message from Vehicle A can conceivably be communicated from A to B, then $\mathrm{B}$ to $\mathrm{C}$. If time were not an issue, then each vehicle may obtain the information it requests. On the other hand, notice that the value of these messages decays quickly: vehicle characteristics at any given point are irrelevant as time passes, and the group of adjacent vehicles also changes rapidly. So there simply may not be enough time to fulfill each vehicle's request before the information becomes valueless.

If a vehicle cannot get one message, then perhaps it requests another; however, each vehicle is not indifferent between all the messages generated in its vicinity. For example, Vehicle A may wish to acquire the information of Vehicle B (which is closer to A), but

\footnotetext{
${ }^{2}$ From "Routing in Vehicular Ad Hoc Networks: A Survey": "Flooding performs relatively well for a limited small number of nodes and is easy to be implemented. But when the number of nodes in the network increases, the performance drops quickly. The bandwidth requested for one broadcast message transmission can increase exponentially. As each node receives and broadcasts the message almost at the same time, this causes contentions and collisions, broadcast storms and high bandwidth consumption" (Li \& Wang, 2007).

${ }^{3}$ U.S. DOT NHTSA Vehicle Safety Communications-Applications Final Report discusses layers of information exchange in Section 6.3.1 entitled "Multi-Channel Operation": "Under this scheme, BSMs (Basic Safety Messages) and other important messages, including service advertisements, are exchanged on the CCH (Control Channel) (Channel 178) during the CCH interval. During the SCH (Service Channel) interval, a vehicle may tune its DSRC radio away from the $\mathrm{CCH}$ to any of the SCHs to, for example, access a service of interest" (Ahmed-Zaid et al., 2011). In Section 6.2 entitled "Message Composition", the report differentiates between critical information that is regularly broadcast, and other information that may be sent upon request of an application. Also see the SAE Dedicated Short Range Communications Implementation Guide, page. 31 .

${ }^{4}$ Sending targeted messages "show significant improvement of bandwidth utilization with slightly loss of reachability, because the new protocols pick fewer vehicles to re-broadcast the message" (Li \& Wang, 2007).

${ }^{5}$ Alternatively, the computer can attempt to calculate crash probabilities and determine who "should" get what information. Since each agent only submits a request, though, it may be difficult to accurately ascertain such probabilities.
} 
prefers this to acquiring the information of Vehicle $\mathrm{C}$ (which is farther away). Then, we may say that Vehicle $\mathrm{A}$ has a preference relation over the set of all messages which he may obtain. $^{6}$

How shall we coordinate the transfer of information amongst vehicles? One possible solution is to design a resource allocation rule (the recommendation) specifying for each possible situation how agents transfer amongst themselves - we take this approach. We recognize that the actual implementation of the program is a complex combination of technical and institutional effort, but we believe our study sheds light on some economic aspects of the problem.

The Department of Transportation is not the only organization to use information generated by vehicles. Similar safety projects involving government organizations and major car manufacturers have been undertaken in Europe. The European Commission recently contributed over 50 million Euros to three projects involving vehicle communications. ${ }^{7}$ Efforts in the private sector (see Waze, Google's recent billion dollar acquisition) to provide real-time traffic data also depend on users contributing real-time information generated from their commute.

More generally, consider the exchange of electronic files between agents in changing environments. For example, a set of financial institutions exchange real-time financial information, or, mobile phones in short-lived "ad-hoc" networks share information to relieve server load. Each of these applications exhibit the basic properties of the our model. Files that are partially transferred may be corrupt or useless; hence, they are indivisible goods and we refer to them as objects. Objects may be perfectly replicated and transferred from each agent to another. For simplicity, we assume each object is of the same size, and each agent is constrained by the same maximum transfer speed; hence, after a normalization, each object requires one unit of time to transfer from one agent to another. Object transfer may then be represented by rounds during which an agent may simultaneously send one object and receive one object. ${ }^{8}$ During each round, each agent may transfer objects he received in previous rounds. A finite number of rounds is assumed, after which each agent consumes the most preferable object (call this his assignment) of those he has accumulated. ${ }^{9}$ A finite number of rounds reflects the fact that over time, the value of the objects decreases to zero. We study the simple case where each agent's consumption space is the set of all singleton objects. Due to the limited number of rounds, not every agent may receive his most preferred object.

The timing is as follows: 1) a group of agents find themselves at the same physical location, 2) each agent reports his preference to a central computer, 3) according to this report, the computer uses a rule to specify a transfer process and sends this recommendation to the agents, 4) the agents execute this transfer process to arrive at a list of assignments, 5) each agent consumes his assignment, and 6) agents disperse, and a new set of agents

\footnotetext{
${ }^{6}$ In Section 6.1, we consider the case where agents' consumption space is the set of all bundles of messages.

${ }^{7}$ See European Commission Press Release - IP/10/353 24/03/2010. Project websites: www.safespoteu.org, www.coopers-ip.eu, and www.cvisproject.org.

${ }^{8}$ These capacity constraints are reasonable when there are two separate channels: one for sending, and one for receiving.

${ }^{9}$ For a survey of recently proposed routing protocols from an engineering perspective, see Li \& Wang (2007).
} 
convene. ${ }^{10}$ Each agent has preferences over the set of all objects, and it is possible for an agent to "withhold" his object. The number of rounds and the objects contributed determine the possible transfer processes, and hence the set of feasible allocations. Since each agent reports his preference and endowment once before the start, we may approach the scenario as a static resource allocation problem. ${ }^{11}$ We look for rules which specify recommendations for each possible problem.

We focus on several desirable properties of rules and seek to understand their implications. Efficiency says that no resources are wasted. Strategy-proofness says that agents do not benefit from reporting false preferences. If agents incur some unmodelled cost, then to encourage agents to contribute to the system, withholding-proofness requires that each agent is always at least as well off when he contributes all the resources he has (in this case, objects). Next, we propose a fairness axiom based on the concept of reciprocity between agents. Roughly, if $j$ is assigned $i$ 's object, then what $i$ is assigned must be at least as good for him as $j$ 's object. The reciprocity lower bound requires this for every pair of agents. We identify a family of rules satisfying these four properties, and also show that each rule in the family is also immune to preference manipulation by groups (Theorem 1). If we strengthen withholding-proofness to its group analog, then we arrive at impossibility (Theorem 2).

We consider two natural extensions of the model. In the first, each agent owns and consumes bundles of objects. In the second, agents live in a network, and may only transfer to agents to which they are connected. Unfortunately, the positive results of the simple model do not hold. The four properties considered in Theorem 1 are not compatible when agents are endowed with and consume bundles (Theorem 3). When agents live in a network, we show that efficiency is compatible with strategy-proofness, but not with either withholdingproofness or the reciprocity lower bound (Theorem 4).

\subsection{Related Literature}

In a model without replicability, when each agent owns a different object, consumes only one object, and has a strict preference relation over the objects, a unique core allocation exists; Gale's Top Trading Cycles (TTC) algorithm identifies this allocation (Shapley \& Scarf, 1974). It turns out that the only efficient, strategy-proof, and individually rational rule is the one obtained by running TTC for each economy (Ma, 1994; Anno, 2015). In the more general case where each agent may or may not own an object, and, in addition there are some publicly owned objects, a modification of TTC identifies an efficient, strategy-proof, and individually rational rule (Abdulkadiroglu \& Sönmez, 1999; Sönmez \& Ünver, 2010). Furthermore, in the same environment, the full class of group strategy-proof and efficient rules have been characterized (Pycia \& Ünver, 2015).

Replicable objects are neither fully private nor fully public goods, so we cannot apply

\footnotetext{
${ }^{10}$ The size of 1) the agent's report to the central computer (a simply ranking over objects), and 2) the computer's recommendation message are assumed to be small compared to the size of messages that contain safety information. Indeed, this fits with the proposal that the secondary layer of information exchange contains longer messages. The additional time needed to coordinate messages may be seen as the price of coordination.

${ }^{11}$ We imagine a scenario in which every second (or less), the resource allocation rule coordinates for the set of present agents.
} 
previously established techniques. The consumption of an object by one agent does not 1) preclude other agents from consuming the same object, or 2) imply all other agents must consume the object. However, the limited number of rounds available to transfer does impose an upper bound on the number of agents who may be assigned an object. Hence, there is a sense in which an object in our environment lies "in between" a private and public good. Furthermore, our model also differs from the case of production: replication and transfer requires time and the object as inputs, but agents do not consume time, and an agent replicating and transferring the object also keeps a copy of the object for himself (contrary to the standard definition of an input). Although our environment is new, the algorithm identifying our family of rules uses TTC as a key component.

The property of withholding-proofness is a central incentive compatibility requirement in applications where agents may replicate objects (Adar \& Huberman, 2000; Feldman \& Chuang, 2005). Previous studies of standard economic environments show that the property is demanding. In the classic exchange economy with divisible goods, efficiency, individual rationality, and withholding-proofness are incompatible (Postlewaite, 1979). In economies with one divisible private good and one fully public good, the Lindahl rule is not withholding-proof (Thomson, 1979; 2010). When each agent owns different objects, and consume bundles of objects, 1) efficiency and withholding-proofness are incompatible and 2) efficiency, individual rationality, and strategy-proofness are incompatible (Atlamaz \& Klaus, 2007; Sönmez, 1999). We extend the analysis of withholding-proofness to an environment with replicable objects, and show, surprisingly, that it is compatible with several desirable criteria. The crucial difference is that private objects are rival, while objects in our environment exhibit some nonrivalry.

\section{Model}

Let $\mathcal{O}$ be the non-empty finite set of objects (electronic messages with a particular vehicle's real-time characteristics) with generic elements $a, b, c, \ldots ; \mathcal{O}$ includes the null object $\emptyset$. Let $N=\{1, \ldots, n\}$ be the set of agents (vehicles). The consumption space of each agent is $\mathcal{O}$.

Each $i \in N$ initially owns one object $\Omega_{i} \in \mathcal{O} \backslash \emptyset$, where for each $j \in N \backslash\{i\}, \Omega_{i} \neq \Omega_{j}{ }^{12}$ Additionally, each $i \in N$ may either 1) report that he owns $\Omega_{i}$, or 2) "withhold" his resource by reporting that he has nothing, $\Omega_{i}^{\prime}=\emptyset$. Let $\mathcal{O}^{N}$ be the set of all possible profiles of reported endowments.

Let $\mathcal{R}$ be the set of strict preference relations ${ }^{13}$ over $\mathcal{O}$, and $R=\left\{R_{i}\right\}_{i \in N} \in \mathcal{R}^{N}$ denote a profile of preferences. For each $R_{i} \in \mathcal{R}$, let $P_{i}$ be the strict part of $R_{i}$.

A technology is available for $T=2$ rounds for agents to replicate and transfer objects to each other. ${ }^{14}$ A transfer process $t$ specifies for each round, and each agent 1 ) what object

\footnotetext{
${ }^{12}$ The results do not change if we allow agents to initially own nothing.

${ }^{13}$ Complete, transitive, anti-symmetric orders.

${ }^{14}$ After, say, 5 seconds an electronic message with a vehicle's real-time characteristics becomes useless (uniformly for everyone); hence, the fact that $T$ is "small" is consistent with our motivating application. Also, the problem of finding the smallest $T$ such that for each economy, each agent may receive each object he desires is closely related to the "Gossip Problem" considered in discrete mathematics (Baker \& Shostak, 1971).
} 
he transfers, and 2) to whom he transfers. Each transfer process $t$ satisfies the following conditions:

T1 In the first round, each agent may replicate one object in his endowment, and transfer this copy of the object to another agent.

T2 In each subsequent round, each agent may replicate either one object in his endowment, or one of the objects he received in a previous round, and transfer this copy of the object to another agent.

T3 In each round, each agent may transfer and receive only one object. This implies an agent may receive up to $T$ objects.

Consumption occurs after all transfers are complete. We study the $T=2$ case as it is the simplest case where replicability is possible; however, we also note instances where results do not rely on this assumption. ${ }^{15}$

The set of agents and the number of available rounds are fixed. An economy $E$ consists of a preference profile $R \in \mathcal{R}^{N}$ and an endowment profile $\Omega \in \mathcal{O}^{N}$; note that we include the possibility that some agents withhold their respective objects. Let $\mathcal{E}=\mathcal{R}^{N} \times \mathcal{O}^{N}$ be the set of all economies.

For each transfer process $t$, a pre-allocation $y(t)$ is a list specifying for each $i$ the set of objects in $\mathcal{O}$ he has accumulated when applying the process $t$. Furthermore, let $\hat{\Omega}_{i} \neq \emptyset$ be $i$ 's initially owned object; then, whether or not he reports $\Omega_{i}=\emptyset, \hat{\Omega}_{i} \in y_{i}(t)$. The interpretation is that even if he does not contribute it, $i$ always keeps a copy of his own object. ${ }^{16}$ An allocation $z$ is a list specifying for each agent $i$ an object in $\mathcal{O}$. An allocation $z$ is feasible for $E$ if there exists a transfer process $t$ such that for each $i \in N, z_{i} \in y_{i}(t)$. For each $E \in \mathcal{E}$, let $Z(E)$ be the set of feasible allocations for $E$.

A rule $\varphi: \mathcal{E} \rightarrow \bigcup_{E \in \mathcal{E}} Z(E)$ assigns each economy a feasible allocation for that economy.

\begin{tabular}{ccccccccc}
$R_{1}$ & $R_{2}$ & $R_{3}$ & $R_{4}$ & $R_{5}$ & $R_{6}$ & & Round 1 & Round 2 \\
\hline$c$ & $a$ & $a$ & $a$ & $a$ & $\emptyset$ & & $1 \stackrel{a}{\rightarrow} 2$ & $1 \stackrel{a}{\rightarrow} 3$ \\
$b$ & $d$ & $c$ & $d$ & $b$ & $f$ & & $2 \stackrel{b}{\rightarrow} 5$ & $2 \stackrel{a}{\rightarrow} 4$ \\
$d$ & $\emptyset$ & $\emptyset$ & $\emptyset$ & $e$ & $\vdots$ & & & $5 \stackrel{b}{\rightarrow} 6$ \\
$e$ & $b$ & $\vdots$ & $\vdots$ & $\vdots$ & & & $3 \stackrel{c}{\rightarrow} 1$ & $4 \stackrel{d}{\rightarrow} 1$
\end{tabular}

\footnotetext{
${ }^{15}$ Clearly there are more general ways to model transfer processes. For example, we may allow each agent to simultaneously transfer to other agents, have a capacity constraint on the total "outflow" rate, have a capacity constraint on the total "inflow" rate, transfer "pieces" instead of objects, and so forth. Our assumptions on the transfer process can roughly be seen as the special case when object size and capacity constraints are uniform across agents, and there is a total of $T=2 r$ time (where $r$ is the minimum time it takes to transfer one object).

${ }^{16}$ In a pre-allocation $y(t)$, an agent possesses up to $T+1$ objects (including his own); however, each agent only consumes his most preferred object in his pre-allocation bundle. In Section 6, we also consider the case where each agent consumes each object in his pre-allocation.
} 
Figure 1: An economy, a transfer process, and feasible allocations. An economy $E=(R, \Omega) \in \mathcal{E}$ is represented on the left. The column $R_{1}$ reads " $c P_{1} b P_{1} d P_{1}$ e $P_{1} a P_{1} \emptyset$ " with each other object ranked below, and the box symbol $a$ indicates $\Omega_{1}=a$. The same holds for each other column $R_{i}$. For brevity, when representing $R_{i}$, we may truncate $i$ 's preference relation after $\Omega_{i}$ as in the column for $R_{2}, R_{5}$, and $R_{6}$. On the right, a transfer process is summarized. The column $\mathrm{R} 1$ indicates transfers occuring in the first round, and similarly for $\mathrm{R} 2$. The symbol $1 \stackrel{a}{\rightarrow} 2$ reads " 1 replicates and transfers object $a$ to 2". Conditions T1-T3 are satisfied. The transfer process results in preallocation $(a c d, b a, c a, d a, e b, f b)$, and e.g. implies the allocations $(c, a, a, a, b, b)$ and $(d, b, a, a, b, \emptyset)$ are feasible.

A formal treatment of the feasible set is provided in Supplementary Appendix.

\section{Axioms}

In this section, we list desirable properties of rules.

The first says that there is no alternative that would make some agents better of without making others worse off. An allocation $z$ is Pareto efficient for $E=(R, \Omega) \in \mathcal{E}$ if there is no $z^{\prime} \in Z(E)$ s.t.

1. for each $i \in N, z_{i}^{\prime} R_{i} z_{i}$, and

2. for at least one $i \in N, z_{i}^{\prime} P_{i} z_{i}$.

Efficiency: For each $E=(R, \Omega) \in \mathcal{E}, \varphi(E)$ is Pareto efficient for $E$.

Next, we introduce incentive compatibility constraints.

The key property in designing resource allocation rules is the prevention of manipulation by unilateral misreporting of preferences. ${ }^{17}$ A stronger property of non-manipulation of the rule by group misrepresentation of preferences follows. These conditions ensure the designer elicits the "true" preference profile; without this property, efficiency and other fairness criteria are evaluated from the standpoint of a "false" preference profile.

Strategy-proofness: For each $E=(R, \Omega) \in \mathcal{E}$, each $i \in N$, and each $R_{i}^{\prime} \in \mathcal{R}$,

$$
\varphi_{i}(E) R_{i} \varphi_{i}\left(R_{i}^{\prime}, R_{-i}, \Omega\right)
$$

Group strategy-proofness: For each $E=(R, \Omega) \in \mathcal{E}$, there is no $S \subseteq N$, and $R_{S}^{\prime} \in \mathcal{R}^{S}$ such that for each $i \in S$,

$$
\varphi_{i}\left(R_{S}^{\prime}, R_{-S}, \Omega\right) R_{i} \varphi_{i}(E)
$$

and for some $j \in S$,

\footnotetext{
${ }^{17}$ Notice that an agent misreports by lying about his preference, not about the information he transfers (e.g. location, speed, etc.).
} 


$$
\varphi_{j}\left(R_{S}^{\prime}, R_{-S}, \Omega\right) P_{j} \varphi_{j}(E)
$$

The next axiom simultaneously addresses the manipulability of a rule and the incentivization for agents to fully contribute their resources. It requires that each agent may never be better off by withholding some of his endowment. In our application, withholding means an agent may somehow "turn off" the machine that allows him to send information, while keeping on the machine that allows him to receive. We also mention its role in combating free-riding below. When an agent only owns one object and withholds, then we simply write that his endowment is $\emptyset$. A stronger version involving manipulation of the rule by groups follows.

Withholding-proofness: For each $E=(R, \Omega) \in \mathcal{E}$, each $i \in N$, and each $\Omega_{i}^{\prime} \subseteq \Omega_{i}$

$$
\varphi_{i}(E) R_{i} \varphi_{i}\left(R, \Omega_{i}^{\prime}, \Omega_{-i}\right)
$$

Group withholding-proofness: For each $E=(R, \Omega) \in \mathcal{E}$, there is no $S \subseteq N$ and $\Omega_{S}^{\prime} \in \mathcal{R}^{S}$ s.t. for each $i \in S, \Omega_{i}^{\prime} \subseteq \Omega_{i}$,

$$
\varphi_{i}\left(R, \Omega_{S}^{\prime}, \Omega_{-S}\right) R_{i} \varphi_{i}(R, \Omega)
$$

and for some $j \in S$,

$$
\varphi_{j}\left(R, \Omega_{S}^{\prime}, \Omega_{-S}\right) P_{j} \varphi_{j}(E) .
$$

Let non-manipulability be the property which states that each agent is always at least as well off when telling the truth than when he jointly 1) lies about his preference, and 2) withholds some resources. The conjunction of withholding-proofness and strategy-proofness imply non-manipulability: For each $E \in \mathcal{E}$, each $i \in N$, each $R_{i}^{\prime} \in \mathcal{R}$, and each $\Omega_{i}^{\prime} \subseteq \Omega_{i}$, $\varphi_{i}(R, \Omega) R_{i} \varphi\left(R, \Omega_{i}^{\prime}, \Omega_{-i}\right) R_{i} \varphi_{i}\left(R_{i}^{\prime}, R_{-i}, \Omega_{i}^{\prime}, \Omega_{-i}\right)$.

Participation constraints are standard in the design of rules: no agent should be worse off than how he started. For each $\Omega \in \mathcal{O}^{N}$, let $\hat{\Omega}$ be the "true" endowment profile-no agent withholds his object.

Individual Rationality For each $E=(R, \Omega) \in \mathcal{E}$, and each $i \in N, \varphi_{i}(E) R_{i} \hat{\Omega}_{i}$.

For each $E \in \mathcal{E}$, and each $z \in Z(E)$, notice that if $z$ does not satisfy individual rationality for $S \subseteq N$ at $E$, there is $z^{\prime}=\left(z_{S}^{\prime}=\Omega_{S}, z_{-i}\right) \in Z(E)$ that Pareto-dominates $z$ and satisfies the property at $E$. This follows from the fact that each agent "keeps a copy" of his own object. Hence, one interesting feature of our environment not present in the standard object re-allocation problem is that efficiency implies individual rationality.

Next, we wish to require some sort of fairness. The first property is well-known: no agent prefers another agent's assignment to his own. 
No-envy For each $E=(R, \Omega) \in \mathcal{E}$, and each $i, j \in N, \varphi_{i}(E) R_{i} \varphi_{j}(E)$.

Efficiency and no-envy are incompatible, as is the case in many environments with discrete resources and deterministic rules. For example, in the economy $E$ in Figure 2 below, each rule satisfying efficiency assigns $a$ to 3 agents (this is the maximum number of agents that can receive $a$ ) in the set $\{2,3,4,5\}$, leaving one agent in the set envying the others. The same issue occurs if we define a weaker property based on treating agents equally if they have the same preference. Hence, we look for alternative fairness criteria.

One issue with no-envy (and the weaker equal treatment property) is that the agents' contributions are not taken into account. What if $\Omega_{i}$ is assigned to several agents, whereas $\Omega_{j}$ is not desired by anyone? Would one find compelling a system where others received your information, but you could not get theirs? It seems intuitive and minimally fair to recognize this asymmetry.

Furthermore, in real-life "peer-to-peer" systems of electronic resource exchange, freeriding is cited as a frequent and prevalent frustration - in one popular systems, $70 \%$ of users did not contribute (Adar \& Huberman, 2000). A growing literature (featuring contributions by both economists and computer scientists) demonstrates that incentive compatibility considerations and reciprocal arrangements help to combat this problem (Feldman \& Chuang, 2005). Here, withholding-proofness and the following property formally play these roles.

Motivated by the points above, we propose a fairness axiom that embodies the concept of reciprocity between agents: if you help me, then I should help you (if you wish). More formally, if agent $j$ is assigned an object $a$ which originally belonged to $i$, then what $i$ is assigned should be at least as good as $j$ 's endowment. The axiom requires that this condition hold for each pair of agents.

Reciprocity lower bound: For each $E=(R, \Omega) \in \mathcal{E}$, there is no $i, j \in N$ such that $\varphi_{j}(E)=\Omega_{i}$, and $\Omega_{j} P_{i} \varphi_{i}(E)$.

\begin{tabular}{|c|c|c|c|c|c|c|c|c|c|c|c|c|}
\hline$R_{1}$ & $R_{2}$ & $R_{3}$ & $R_{4}$ & $R_{5}$ & $R_{1}$ & $R_{2}$ & $R_{3}$ & $R_{4}$ & $R_{5}$ & $R_{6}$ & $R_{7}$ & $R_{8}$ \\
\hline$c$ & $a$ & $a$ & $a$ & $a$ & $b$ & $a$ & $a$ & $a$ & $a$ & $b$ & $b$ & $b$ \\
\hline$b$ & $b$ & $c$ & $d$ & $e$ & $a$ & & & & $b$ & & & \\
\hline
\end{tabular}

Figure 2: Let $E \in \mathcal{E}$ be as on the left above. If $z=(b, a, a, a, e)$, then notice that $z_{3}=a=\Omega_{1}$, and $\Omega_{3}=c P_{1} b=z_{1}$; so $z$ violates the reciprocity lower bound (but satisfies no-envy). If 2 is assigned $a$, then the property requires that 1 is assigned $b$ or $c$. If 3 is assigned $a$, then the property requires that 1 is assigned $c$. If $z=(c, a, a, a, e)$, then $z$ violates no-envy but satisfies the reciprocity lower bound. The profile on the right pertains to the discussion of the core.

We formally discuss its relation to other concepts.

It has zero efficiency content: A rule that assigns $(\emptyset, \ldots, \emptyset)$ for each economy satisfies the reciprocity lower bound. Also, no-envy and the reciprocity lower bound are logically independent as the examples in Figure 2 show. In contrast to no-envy though, efficiency and the reciprocity lower bound are compatible.

Although the property is reminiscent of the core constraints, notice that it does not establish the right of an agent to select whom to trade with. For example, in Figure 2 
in the right economy, $z=(a, a, a, a, b, b, b, b)$ is an allocation that may be recommended by a rule satisfying efficiency and the reciprocity lower bound; however, if 1 and 5 had a choice, they would choose to trade with each other. This clearly violates core considerations. Furthermore, as we see below, allocations recommended by a rule satisfying the reciprocity lower bound may fall outside of an even weaker version of the core.

Formally, an allocation $z \in Z(E)$ is in the core of $\boldsymbol{E}$ if there is no $S \subseteq N$ and $\left(z_{S}^{\prime}, z_{-S}\right) \in$ $Z(E)$ such that for each $i \in S, z_{i}^{\prime} \in \Omega_{S}, z_{i}^{\prime} R_{i} z_{i}$, and for some $j \in S, z_{j}^{\prime} P_{j} z_{j}$. First, the core constraints contain statements for all possible groups of agents, while the reciprocity lower bound is simply pairwise. Second, the core may be empty: Let $E \in \mathcal{E}$ be as in Figure 2, except 1 top-ranks his own object. There are four efficient allocations $z^{1}=(a, a, a, a, e), z^{2}=$ $(a, a, a, d, a),(a, a, c, a, a)$, and $(a, b, a, a, a)$. The first allocation is not in the core for $E$; let $S=\{1,2,3,5\}$ and $z^{2}$. A symmetric reasoning holds for the other three allocations. Contrast this to the fact that for each economy $E$, the set of allocations that may be recommended by a rule that satisfies the reciprocity lower bound is non-empty. Third, each selection from the core correspondence satisfies the reciprocity lower bound: Let $\varphi(E)=z$ be in the core of $E$, and $i, j \in N$ be such that $z_{j}=\Omega_{i}$. Let $S=\{i, j\}$, then there is $z^{\prime} \in Z(E)$ such that $z_{i}^{\prime}=\Omega_{j}$ and $z_{j}^{\prime}=\Omega_{i}$-implying $z_{i} R_{i} \Omega_{j}$. Lastly, there are rules that satisfy efficiency and the reciprocity lower bound, but are not selections from the core. As in a previous example, let $\varphi$ be such that for $E$ in Figure 2 on the right, $\varphi(E)=(a, a, a, a, b, b, b, b)$. Notice that $\varphi(E)$ is not in the core of $E$, but $\varphi$ may be extended to satisfy the two previous properties. An allocation $z \in Z(E)$ is in the weak core of $\boldsymbol{E}$ if there is no $S \subseteq N$ and $\left(z_{S}^{\prime}, z_{-S}\right) \in Z(E)$ such that for each $i \in S, z_{i}^{\prime} \in \Omega_{S}$ and $z_{i}^{\prime} P_{i} z_{i}$. The weak core is always non-empty (our main rules serve as examples), but selections from the weak core correspondence may violate both efficiency and the reciprocity lower bound: in the economy $E$ in Figure 2 on the left, $z=(c, b, c, d, e)$ is in the weak core of $E$, but cannot be the recommendation of a rule satisfying either of the two properties. Also, an allocation that is recommended by a rule satisfying the reciprocity lower bound may fall outside of the weak core: the allocation where

each agent is assigned his endowment satisfies the property, but may not be in the weak core e.g. if one agent top-ranks an object other than his endowment.

\section{Rules}

The family of serial priority rules is central in the study of resource allocation problems. We introduce a second family of rules which are of particular interest.

\subsection{Serial Priority w.r.t. $\sigma$}

Let $\sigma:\{1, \ldots, n\} \rightarrow N$ be an ordering of $N$. For each $E \in \mathcal{E}$, each $Z \subseteq Z(E)$, and each $i \in N$, let $Z_{i} \equiv\left\{x \in \mathcal{O}: \exists z \in Z\right.$ s.t. $\left.x=z_{i}\right\}$ be the assignments which are feasible for $i$ in the set $Z$.

- Let $Z^{1} \equiv\left\{z \in Z(E): z_{\sigma(1)}=\arg \max _{Z_{\sigma(1)}(E)} R_{\sigma(1)}\right\}$.

- Let $Z^{2} \equiv\left\{z \in Z^{1}: z_{\sigma(2)}=\arg \max _{Z_{\sigma(2)}^{1}} R_{\sigma(2)}\right\}$. 
-

- Let $Z^{n} \equiv\left\{z \in Z^{n-1}: z_{\sigma(n)}=\arg \max _{Z_{\sigma(n)}^{n-1}} R_{\sigma(n)}\right\}$.

Then, $S P^{\sigma}(E) \equiv Z^{n}$.

Example Serial Priority is not withholding-proof. Let $\sigma(i)=i$, and $j=12$. The first two agents choose $\Omega_{13}$; the next three, $\Omega_{1}$; the next three, $\Omega_{2}$; the next three, $\Omega_{12}$. Next is agent 12 who top-ranks $\Omega_{13}$. Notice that it is not feasible for agent 12 to be assigned $\Omega_{13}$ given the previous assignments. However, if agent 12 withholds, and the three agents who chose $\Omega_{12}$ before now choose their own endowments, it is feasible for agent 13 to receive $\Omega_{12}$.

This is surprising, given that Serial Priority is seen as a "rigid" rule satisfying strong incentive compatibility requirements. It indicates that manipulation by endowments is indeed of a different character than manipulation by preference misrepresentation.

\subsection{Top Trading Cycles with Serial Inheritance w.r.t. $\sigma$}

For each object $a \in \mathcal{O}$, let $i_{a}$ be the owner of $a$. For each $E \in \mathcal{E}$, and $z \in Z(E)$, an object $a \in \mathcal{O}$ is attainable given $z$ and $E$ if

1. in $E, i_{a}$ reports that $\Omega_{i_{a}}=a$, as opposed to $\Omega_{i_{a}}^{\prime}=\emptyset$,

2. for each $j \in\left\{\ell \in N: z_{\ell}=\emptyset\right\}, \exists z^{\prime} \in Z(E)$ s.t. for each $i \in\left\{\ell \in N: z_{\ell} \neq \emptyset\right\}, z_{i}^{\prime}=z_{i}$, and $z_{j}^{\prime}=a$.

The first condition ensures that the owner $i_{a}$ of $a$ does not withhold $a$. Consider an agent $j$ who is assigned $\emptyset$ at $z$, and an allocation $z^{\prime}$ which is the same as $z$, with the exception that $j$ is assigned $a$. The second condition requires that $z^{\prime}$ is feasible given $E$, no matter which unassigned agent $j$ we select. ${ }^{18}$

Let $\sigma:\{1, \ldots, n\} \rightarrow N$ be an ordering of $N$, and $x^{0}=(\emptyset, \ldots, \emptyset)$. The top trading cycles with serial inheritance w.r.t. $\boldsymbol{\sigma}, \boldsymbol{T} \boldsymbol{T} \boldsymbol{C}-\boldsymbol{S} \boldsymbol{I}^{\sigma}$, rule is given by an algorithm as follows:

Step 1: Construct a graph where each agent $i \in N$, and each attainable object given $x^{0}$ and $E$ is a node. Each object (besides $\emptyset$ ) points to its owner (forming a directed edge). Each agent points to his most preferred object. If an agent points to $\emptyset$, then $\emptyset$ points back to him. There is at least one cycle. Let $x^{1}$ be such that 1) for each agent $i$ who is in a cycle, $x_{i}^{1}$ is the object he points to, and 2) for each other agent, $x_{i}^{1}=\emptyset$. Each agent assigned an object leaves. Each agent who was in a cycle leaves.

\footnotetext{
${ }^{18}$ We may weaken the definition of attainability to only require that at least some agent $j$ can receive the object $a$. In the following algorithm, for each allocation that can be reached after each Step, the two definitions are equivalent. However, in general this weaker version does not imply the stated version (see Section 6.3).
} 
Step $k$ : If there are no remaining agents, then let $\varphi^{\sigma}(E)=x^{k-1}$ and terminate. Construct a graph where each remaining agent, and each object attainable given $x^{k-1}$ and $E$ is a node. Each present object (besides $\emptyset$ ) points to its owner if he is remaining, otherwise the earliest-priority remaining agent with respect to $\sigma$. Each remaining agent points to his most preferred present object. If an agent points to $\emptyset$, then $\emptyset$ points back to him. There is at least one cycle. Let $x^{k}$ be such that 1 ) for each agent $i$ who has left before Step $k, x_{i}^{k}=x_{i}^{k-1}, 2$ ) for each agent $i$ who is in a cycle, $x_{i}^{k}$ is the object he points to, and 3) for each other agent, $x_{i}^{k}=\emptyset$. Each agent who was in a cycle in this Step leaves.

$T T C-S I^{\sigma}$ is a modification of TTC in that between each step of assigning objects along cycles, there is an additional process of determining the attainable objects. For $T=2$, this process is $O(n \log (n)) .{ }^{19}$ Since there are at most $n$ steps of assigning cycles, checking attainability adds $O\left(n^{2} \log (n)\right)$ additional operations to TTC. Since TTC is polynomial time, $T T C-S I^{\sigma}$ is as well.

Example Let $E=(R, \Omega) \in \mathcal{E}$ be as below, and for each $i \in N, \sigma(i)=i$.

\begin{tabular}{ccccc}
$R_{1}$ & $R_{2}$ & $R_{3}$ & $R_{4}$ & $R_{5}$ \\
\hline$b$ & $a$ & $a$ & $a$ & $a$ \\
$a$ & $b$ & $c$ & $d$ & $e$
\end{tabular}

Step 1: Agents 1 and 2 form the only cycle. Then, $x^{1}=(b, a, \emptyset, \emptyset, \emptyset)$.

Step 2: Notice $a$ and $b$ are attainable given $x^{1}$ under $E$. For example, the allocation $y=(b, a, a, \emptyset, \emptyset)$ "adds" to the allocation $x^{1}$ by assigning agent 3 object $a$. Thus, $y$ is feasible as there is a transfer process (shown below) which results in $y$.

\begin{tabular}{cc} 
Round 1 & Round 2 \\
\hline $1 \stackrel{a}{\rightarrow} 2$ & $1 \stackrel{a}{\rightarrow} 3$ \\
$2 \stackrel{b}{\rightarrow} 1$ &
\end{tabular}

Objects $a$ and $b$ point to the earliest-remaining priority agent 3 . Since all agents point to $a$, but $a$ points to 3 , the only cycle is with agent 3 . Then, $x^{2}=(b, a, a, \emptyset, \emptyset)$.

Step 3: Objects $a$ and $b$ are attainable given $x^{2}$ under $E$. The only cycle is with agent 4 , and $x^{3}=(b, a, a, a, \emptyset)$.

Step 4: Object $a$ is not attainable given $x^{3}$ under $E$, so it is removed from the algorithm; $b$ is still attainable given $x^{3}$ under $E$, and so points at 5 . Since 5's most preferred object is $e$, he points to $e$ and forms a cycle with $e$. Then, $x^{4}=(b, a, a, a, e)$.

Step 5: No agents remain, so $T T C-S I^{\sigma}(E)=x^{4}$.

\footnotetext{
${ }^{19}$ This calculation is performed in the Supplementary Appendix.
} 


\section{Results}

\begin{tabular}{|r|c|c|}
\hline & SP $^{\sigma}$ & TTC $-S I^{\sigma}$ \\
\hline \hline Efficiency & + & + \\
\hline Strategy-proofness & + & + \\
\hline Group Strategy-proofness & + & + \\
\hline Withholding-proofness & - & + \\
\hline Group Withholding-proofness & - & - \\
\hline Reciprocity Lower Bound & - & + \\
\hline
\end{tabular}

Theorem $1 T T C-S I^{\sigma}$ satisfies efficiency, group strategy-proofness, withholding-proofness, and the reciprocity lower bound.

This result is encouraging, since very few economic environments allow for the simultaneity of such strong incentive compatibility, efficiency, and fairness notions. Furthermore, the structure of algorithm is rather straightforward; the familiar "trade along cycles" intuition of TTC comprises a key component of each step. Can we include a more flexibility in the inheritance structure rather than having one agent inherit all the attainable objects? We discuss this possibility in Section 6.3. In Appendix 2, we show that the properties are independent.

Let $\varphi \equiv T T C-S I^{\sigma}$. For each $E \in \mathcal{E}$, let $\bar{k}(E)$ be the last step of the algorithm under $E$. For each $E \in \mathcal{E}$, and $k \in\{1, \ldots, \bar{k}(E)\}$, "Step $k$ under $E$ " refers to the $k^{\text {th }}$ step of the algorithm run for economy $E$, and " $x^{k}$ under $E$ " refers to current allocation at the $k^{\text {th }}$ step of the algorithm run for economy $E$. Let $V$ be the step under $E$ at which $i$ receives his assignment. For the sake of brevity, " $i$ points to $j$ " means $i$ points to an object which points to $j$. A cycle is a set of agents $i_{1}, \ldots, i_{m}$ s.t. $i_{1}$ points to $i_{2}$ points to... $i_{m}$ points to $i_{1}$.

\section{Proof of Theorem 1:}

Efficiency: Consider $E \in \mathcal{E}$. For each $k \in\{1, \ldots, \bar{k}(E)\}$, let $S^{k}$ be the set of agents at Step $k$ who are assigned an object. Let $\tau$ be the ordering of $N$ as follows: the first $\left|S^{1}\right|$ agents in $\tau$ comprise of agents in $S^{1}$, the next $\left|S^{2}\right|$ agents in $\tau$ comprise of agents in $S^{2}, \ldots$ and so on. Then, $\varphi(E)=S P^{\tau}(E)$. Since $S P^{\tau}(E)$ is efficient for $E, \varphi(E)$ is efficient for $E$.

Group strategy-proofness: In this setting, strategy-proofness and non-bossiness imply group strategy-proofness (Pápai, 2000) ${ }^{20}$, we will show each of these two properties are satisfied. The proof for strategy-proofness is similar to (Abdulkadiroğlu \& Sönmez, 1999). The proof for non-bossiness and withholding-proofness are provided in Appendix 1.

\footnotetext{
${ }^{20}$ Although this paper considers a different model, the proof for this statement holds in ours as well.
} 
Lemma 1 For each $E \in \mathcal{E}$, and each $k \in\{2, \ldots, \bar{k}(E)\}$, the set of attainable objects given $x^{k}$ under $E$ is contained in the set of attainable objects given $x^{k-1}$ under $E$. More generally, for each $E \in \mathcal{E}$, if $x, x^{\prime} \in Z(E)$ and for each $i \in N$ s.t. $x_{i} \neq \emptyset, x_{i}=x_{i}^{\prime}$, then the set of attainable objects given $x^{\prime}$ is contained in the set of attainable objects given $x$.

Proof: Let $a \in \mathcal{O}$ be attainable given $x^{\prime}$ and $E$. That is,

1. $\exists i \in N$ s.t. $\Omega_{i}=a$, and

2. $\exists z^{\prime} \in Z(E)$ and $j \in\left\{\ell \in N: x_{\ell}^{\prime}=\emptyset\right\}$ s.t. for each $i \in\left\{\ell \in N: x_{\ell}^{\prime} \neq \emptyset\right\}, z_{i}^{\prime}=x_{i}^{\prime}$, and $z_{j}^{\prime}=a$.

This means there is $t^{\prime}$ such that for each $i \in N, z_{i}^{\prime} \in y_{i}\left(t^{\prime}\right)$. Let $z$ be an allocation such that for each $h \in\left\{\ell \in N: x_{\ell}=\emptyset\right\}, z_{h}=\emptyset, i \in\left\{\ell \in N: x_{\ell} \neq \emptyset\right\}, z_{i}=x_{i}$, and $z_{j}=a$. Since for each $i \in N, z_{i} \in y_{i}\left(t^{\prime}\right), z \in Z(E)$. This implies $a$ is attainable given $x$ and $E$.

Strategy-proofness: Let $E \in \mathcal{E}$ and $i \in N$. Let $V^{\prime}$ be the Step at which $i$ receives his assignment under $E^{\prime} \equiv\left(R_{i}^{\prime}, R_{-i}, \Omega\right) \in \mathcal{E}$. If $\varphi_{i}(E)=\varphi_{i}\left(E^{\prime}\right)$, then we are done. So let $\varphi_{i}(E) \neq \varphi_{i}\left(E^{\prime}\right)$.

Case 1: $V^{\prime} \leq V$. Allocations $x^{1}, \ldots, x^{V^{\prime}-1}$ under $E$ and $E^{\prime}$ are the same. By definition, $i$ is in a cycle $S$ at Step $V^{\prime}$ under $E^{\prime}$. Hence, at Step $V^{\prime}, \ldots, V$ under $E$, each agent in the cycle $S \backslash i$ points to the same object, implying $\varphi(E) P_{i} \varphi\left(E^{\prime}\right)$.

Case 2: $V^{\prime}>V$. At Step $V^{\prime}$ under $E^{\prime}, i$ points to an object $a \in \mathcal{O} \cup \emptyset$. By definition, $a$ is attainable given $x^{V^{\prime}-1}$ under $E^{\prime}$. Suppose $a R_{i} \varphi_{i}(E)$. By assumption of $\varphi_{i}(E) \neq \varphi_{i}\left(E^{\prime}\right)$, $a P_{i} \varphi_{i}(E)$. Steps $1, \ldots, V-1$ under $E$ and $E^{\prime}$ are the same, implying for $x^{V-1}$ under $E$ and $x^{V^{\prime}}$ under $E^{\prime}$, and each $i \in N$ s.t. $x_{i}^{V-1} \neq \emptyset, x_{i}^{V-1}=x_{i}^{V^{\prime}}$. Hence, by Lemma 1 , the set of attainable objects given $x^{V^{\prime}-1}$ is contained in the set of attainable objects given $x^{V-1}$. Since $a$ is attainable given $x^{V^{\prime}-1}$ under $E^{\prime}, a$ is attainable given $x^{V-1}$ under $E$. Hence, at Step $V$ under $E$, $i$ points to $a \neq \varphi_{i}(E)$, contradicting the definition of the rule.

Reciprocity Lower Bound: Let $J$ be the set of $j \in N$ s.t. there is a step at which $j$ points to $\Omega_{i}$. For each $j \in J$, let $k^{j}$ be the first step at which $j$ points to $i$. If $k^{j} \leq V$, then $j$ still points to $i$ until Step $V$. If $k^{j}>V$, then by definition, $j$ has not been assigned an object at Step $V$. Hence, for each $j \in J, \Omega_{j}$ is attainable given $x^{V-1}$. By definition, $i$ is assigned the object he points to at Step $V$, implying that for each $j \in J, \varphi_{i}(E) R_{i} \Omega_{j}$.

Notice that the statements for efficiency, strategy-proofness, and the reciprocity lower bound also hold for the case when $T>2$.

These four axioms do not characterize the $T T C-S I^{\sigma}$ family of rules - we provide an example in Appendix 2 of a rule outside this family. Does a strengthening of withholdingproofness to its group version brings us closer? Unfortunately, such a strengthening ends in incompatibility.

Theorem 2 No rule satisfies efficiency, group strategy-proofness, group withholding-proofness, and the reciprocity lower bound. 
Proof of Theorem 2: $\quad$ Let $\varphi$ be a rule satisfying efficiency, group strategy-proofness, group withholding-proofness, and the reciprocity lower bound. Let $E \in \mathcal{E}$ be as below. For each $i \in N, \Omega_{i} R_{i} \emptyset$.

\begin{tabular}{cccccccc}
$R_{1}$ & $R_{2}$ & $R_{3}$ & $R_{4}$ & $R_{5}$ & $R_{6}$ & & $R_{3}^{\prime}$ \\
\hline$a$ & $c$ & $a$ & $c$ & $c$ & $c$ & & $a$ \\
& $b$ & $b$ & $d$ & $e$ & $f$ & & $d$ \\
& \multicolumn{1}{c}{$c$} & & & & & $c$
\end{tabular}

By efficiency, $\varphi_{3}(E)=a$. Let $E^{1} \equiv\left(R, \Omega_{1}=\emptyset, \Omega_{-1}\right)$. By efficiency and the reciprocity lower bound, $\varphi_{3}\left(E^{1}\right)=b$ and $\varphi_{2}\left(E^{1}\right)=c$. Hence, by group withholding-proofness, $\varphi_{2}(E)=c$.

Let $E^{2} \equiv\left(R_{3}^{\prime}, R_{-3}, \Omega\right)$ and $E^{2 A} \equiv\left(R_{3}^{\prime}, R_{-3}, \Omega_{1}=\emptyset, \Omega_{-1}\right)$. By efficiency and the reciprocity lower bound, $\varphi_{3}\left(E^{2 A}\right)=d$ and $\varphi_{4}\left(E^{2 A}\right)=c$. Hence, by group withholding-proofness, $\varphi_{4}\left(E^{2}\right)=c$. By group strategy-proofness, $\varphi_{4}(E)=c$. Analagously, $\varphi_{5}(E)=\varphi_{6}(E)=c$. However, this violates feasibility, since $c$ is assigned to $4>2^{T}-1$ agents.

\section{Extensions}

We consider two generalizations of the previous model. In the first, each agent consumes multiple objects, and may own multiple objects. ${ }^{21}$ In the second, agents live in a network represented by a graph, and can only transfer to adjacent agents. The results are more disappointing than those in the simple model.

Next we may wonder if we can define "inheritance trees", and trade along cycles as in (Pápai, 2000). We attempt to construct such a rule, and show it is not well-defined.

\subsection{Agents Consume Bundles}

Let $\mathcal{O}=\{a, b, c, \ldots\}$ be the finite set of objects, and $N=\{1, \ldots, n\}$ be the set of agents. The consumption space of each agent is $2^{\mathcal{O}} \cup \emptyset$. Write the set $\{a, b, c\}$ as $a b c$.

Let $\mathcal{S}$ be the set of strict preference relations over $2^{\mathcal{O}}$. Given $R_{i} \in \mathcal{S}$, an object $x \in \mathcal{O}$ is desirable for $i$ if $x R_{i} \emptyset$; let $D_{i} \subseteq \mathcal{O}$ be the set of desirable objects for $i$. Below we list three conditions on preferences.

Consistency w.r.t. absolute desirability For each $x \in D_{i}$, and each $S \subseteq D_{i} \backslash x, S \cup$ $x R_{i} S$.

Consistency w.r.t. relative desirability For each $x, y \in D_{i}$, and each $S \subseteq D_{i}, x R_{i} y \Longleftrightarrow$ $S \cup x R_{i} S \cup y$.

Consistency w.r.t. undesirability For each $x \in \mathcal{O} \backslash D_{i}$, and each $S \subseteq \mathcal{O}, \emptyset R_{i} S \cup x$.

Let $\mathcal{R} \subset \mathcal{S}$ be the domain of preferences satisfying the three conditions above (also known as responsive preferences (Roth, 1985)). The domain $\mathcal{R}$ excludes preference relations with

\footnotetext{
${ }^{21}$ When objects are fully private goods, efficiency, individual rationality, and strategy-proofness are incompatible (Sönmez, 1999). This result does not hold in our model because individual rationality is trivially satisfied, as agents always keep a copy of their own object.
} 
complementarities - if $a, b, c \in \mathcal{O}$ are such that $a P_{i} b P_{i} c P_{i} \emptyset$, then $a b P_{i} b c$. Let $R=$ $\left\{R_{i}\right\}_{i \in N} \in \mathcal{R}^{N}$ denote a profile of preferences.

The same technology as in the previous section is available. An economy and preallocation are defined as before. However, an allocation is a list specifying for each agent $i$ a set of objects in $\mathcal{O}$. An allocation $z$ is feasible if for each $i \in N$, there is a transfer process $t$ resulting in a pre-allocation $y(t)$ such that $z_{i} \subseteq y_{i}(t)$.

We modify the definition of reciprocity lower bound in this extension. If agent $j$ receives $k$ number of objects which originally came from $i$ 's endowment, then $i$ should have the "right" to any $k$ objects from $j$ 's endowment. If agent $j_{1}$ and $j_{2}$ each receive $k_{1}$ and $k_{2}$ objects which originally came from $i$ 's endowment, then $i$ should have the "right" to any bundle consisting of $k_{1}$ (or fewer) of $j_{1}$ 's endowment and $k_{2}$ (or fewer) of $j_{2}$ 's endowment. A similar statement holds if 3 agents receive $i$ 's objects.

For each $E \in \mathcal{E}$, each $z \in Z(E)$, and each $i, j \in N$, let $k_{j}^{i}(z, E)=\left|\Omega_{i} \cap z_{j}\right|$ and $X_{i}(z, E) \equiv\left\{x \subseteq O:|x| \leq T\right.$ \& for each $\left.j \in N \backslash i,\left|x_{i} \cap \Omega_{j}\right| \leq k_{j}^{i}(z, E)\right\}$. That is, $X_{i}(z, E)$ is the set of bundles $i$ may choose from if he had the right to $k_{j}^{i}(z, E)$ objects from the endowment of $j$. Let $\bar{X}_{i}(z, E) \equiv \Omega_{i} \cup\left\{\arg \max _{X_{i}(z, E)} R_{i}\right\}$.

Reciprocity lower bound: For each $E(R, \Omega) \in \mathcal{E}$, and each $i \in N, \varphi_{i}(E) R_{i} \bar{X}_{i}(\varphi(E), E)$.

In environments where agents are endowed with and consume bundles, and replicability is not possible 1) efficiency and withholding-proofness are incompatible and 2) efficiency, individual rationality, and strategy-proofness are incompatible (Atlamaz \& Klaus, 2007; Sönmez, 1999; Konishi, Quint, \& Wako, 2001).

With replicability, we also see the contrast between the simple and more general case. The four properties that were compatible before, are now incompatible.

Theorem 3 No rule satisfies efficiency, strategy-proofness, withholding-proofness, and the reciprocity lower bound.

Proof of Theorem 3: Let $E=(R, \Omega) \in \mathcal{E}$ be as below. For each $i \in N$, and each $z \subseteq \mathcal{O}$, $z R_{i} \emptyset$.

\begin{tabular}{|c|c|c|c|c|c|}
\hline$R_{1}$ & $R_{2}$ & $R_{3}$ & $R_{4}$ & $R_{4}^{\prime} \quad R_{4}^{\prime \prime}$ & \\
\hline$e$ & $e$ & $g$ & $a$ & $f$ & (abe, cdeb, efgd, gac) \\
\hline$f$ & $b$ & $d$ & $c$ & $c$ & $(a b e f, c d e b, e f g d, g c)$ \\
\hline$a b$ & $c d$ & $e f$ & $f$ & $a$ & (abef,cde, efgd,gac) \\
\hline & & & $g$ & \begin{tabular}{|l|}
$g$ \\
\end{tabular} & \\
\hline
\end{tabular}

The set of allocations satisfying efficiency and the reciprocity lower bound is $\{A, B, C\}$ defined above.

Case 1: $\varphi(E)=A$. Let $\Omega_{1}^{\prime}=a$. By efficiency, $\varphi\left(R, \Omega_{-1}, \Omega_{1}^{\prime}\right)=C$. Agent 1 has incentive to withhold.

Case 2: $\varphi(E)=B$. Let $R_{4}^{\prime}$ be as above. By efficiency and the reciprocity lower bound, $\varphi\left(R_{4}^{\prime}, R_{-4}, \Omega\right)=(a b e, c d e b$, efgd, gcf $)$. Agent 4 has incentive to misreport his preference. 
Case 3: $\varphi(E)=C$. Let $\Omega_{2}^{\prime}=d$, and $E^{3} \equiv\left(R, \Omega_{2}^{\prime}, \Omega_{-2}\right)$. By efficiency and the reciprocity lower bound, $\varphi\left(E^{3}\right)$ is either $D \equiv\left(\right.$ abef, cde, efgd,ga), or $F \equiv(a b e, c d e b$, efgd,gaf $)$. Let $R_{4}^{\prime \prime}$ be as above, and $E^{4} \equiv\left(R_{-4}, R_{4}^{\prime \prime}, \Omega_{-2}, \Omega_{2}^{\prime}\right)$. By efficiency and the reciprocity lower bound, $\varphi\left(E^{4}\right)=\left(\right.$ abe, cdeb, efgd, gaf). Hence, $\varphi\left(E^{3}\right)=F$, otherwise agent 4 may benefit from misreporting his preference. So $\varphi_{2}\left(E^{3}\right) R_{2} \varphi_{2}(E)$, giving agent 2 incentive to withhold.

\subsection{Network Constraints}

Consider the following extension to the model. A network is $G \subseteq N \times N$ s.t.

- for each $g=\left(g_{1}, g_{2}\right) \in G, g_{1} \neq g_{2}$, and

- if $\left(g_{1}, g_{2}\right) \in G$, then $\left(g_{2}, g_{1}\right) \in G$.

Let $\mathcal{G}$ be the set of all networks. For each network $G \in \mathcal{G}$, each agent may only replicate and transfer to adjacent agents in $G$. Hence, the feasibility constraints become:

N1 In the first round, each agent $i$ may replicate one object in his endowment, and transfer this copy to another agent $j$, only if $(i, j) \in G$.

N2 In each subsequent round, each agent $i$ may replicate either one object in his endowment, or one of the objects he received in a previous round, and transfer this copy to another agent $j$, only if $(i, j) \in G$.

N3 In each round, each agent may transfer and receive only one object.

Let $\mathcal{E} \equiv \mathcal{G} \times \mathcal{R}^{N} \times \mathcal{O}^{N}$ be the set of economies. A feasible allocation, and rule are defined as before.

Efficiency and strategy-proofness are compatible-the serial priority rule is an example. However, in a large class of networks, efficiency is compatible with neither withholdingproofness nor the reciprocity lower bound.

A path in $G$ is a sequence of agents $i_{1}, i_{2}, \ldots, i_{m} \in N$ such that for each $k \in\{1, \ldots, m-$ $1\},\left(i_{k}, i_{k+1}\right) \in G$. The length of a path $i_{1}, \ldots, i_{m}$ is $m-1$.

Theorem 4 If there is $i, j \in N$ s.t. $(i, j) \notin G$, and there is only one path of length $T$ from $i$ to $j$, then

1. no rule satisfies efficiency and withholding-proofness, and

2. no rule satisfies efficiency and the reciprocity lower bound.

If there is a pair of agents such that the only path to each other is only by an intermediate agent, then incompatibilities arise. Note that this connectivity property does not preclude the pair of agents from having multiple connecting paths of length $\ell>T$, so large classes of networks satisfy this property. Furthermore, it is clear that $T$ plays an important role in this connectivity condition. Transferring an object from $i$ to $j$ takes exactly $T$ rounds 
and the usage of the path from $i$ to $j$; hence, at each Round, there is agent $k$ on this path is transferring $i$ 's object to the next agent. Such an arrangement unfortunately precludes transferring an object from $j$ to $i$ : if we attempt to do this, then there will be a Round where an agent must simultaneously transfer $\Omega_{i}$ and $\Omega_{j}$ to two different agents.

Theorem 4 is disappointing given that in applications it is very possible agents are network constrained.

Proof of Theorem 4: Let $E=(G, R, \Omega) \in \mathcal{E}$ satisfy the hypothesis above, and $i, \ell, j \in N$ be the path of length $T=2$ from $i$ to $j$. For each $i \in N$, and each $y \in \mathcal{O}, y R_{i} \emptyset$. Let $\left(R_{h}, \Omega_{h}\right)_{h \in\{i, \ell, k\}}$ be as below. Let $R_{-\{i, \ell, k\}}$ be such that

- for each $j \in N \backslash\{i, \ell, k\}$, and each $z \in\{i, \ell, k\}, \emptyset R_{j} \Omega_{z}$, and

- for each $f \in\{i, k\}$, and each $j, h \in N$ s.t. $(j, z),(h, z) \in G$ and $h \neq j, \emptyset R_{j} \Omega_{h}$.

\begin{tabular}{|c|c|c|c|c|}
\hline$R_{i}$ & $R_{\ell}$ & $R_{k}$ & Round 1 & Round 2 \\
\hline$c$ & $a$ & $a$ & $i \stackrel{a}{\rightarrow} \ell$ & \\
\hline$b$ & $c$ & $b$ & $\ell \stackrel{b}{\rightarrow} i$ & $\ell \stackrel{a}{\rightarrow} k$ \\
\hline$a$ & $b$ & \begin{tabular}{|l}
$c$ \\
\end{tabular} & & \\
\hline
\end{tabular}

Let $\varphi_{i \ell k}(E)=\left(\varphi_{i}(E), \varphi_{\ell}(E), \varphi_{k}(E)\right)$. By efficiency, $\varphi_{i \ell k}(E)$ is either 1) $(b, a, a)$, or 2) $(c, c, b)$.

Withholding-proofness:

Case 1: $\varphi_{i \ell k}(E)=(b, a, a)$. Let $E^{\prime}=\left(G, R, \Omega_{i}^{\prime}=\emptyset, \Omega_{-i}\right)$. By efficiency, $\varphi_{i \ell k}\left(E^{\prime}\right)=$ $(c, c, b)$. Hence, $i$ is made better off, in violation of withholding-proofness.

Case 2: $\varphi_{i \ell k}(E)=(c, c, b)$. Let $E^{\prime \prime}=\left(G, R, \Omega_{k}^{\prime}=\emptyset, \Omega_{-k}\right)$. By efficiency, $\varphi_{i \ell k}\left(E^{\prime \prime}\right)=$ $(b, a, a)$. Hence, $k$ is made better off, in violation of withholding-proofness.

Reciprocity lower bound:

Case 1: $\varphi_{i \ell k}(E)=(b, a, a)$. Then, $\bar{X}_{i}(\varphi(E), E)=c R_{i} b=\varphi_{i}(E)$, in violation of reciprocity lower bound.

Case 2: $\varphi_{i \ell k}(E)=(c, c, b)$. Then, $\bar{X}_{k}(\varphi(E), E)=a R_{k} b=\varphi_{k}(E)$, in violation of reciprocity lower bound.

\subsection{Trading Along Cycles with Inheritance Trees}

In this section we ask if we may extend TTC with "inheritance trees" to our setting (Pápai, 2000). We attempt to construct such a rule, and show it is not well-defined. The idea is the following: In the $T T C-S I^{\sigma}$ rules, at each Step a single agent inherits all the attainable objects without an owner. Here, we show that if we allow for several agents to inherit attainable objects, then there is a clash with feasibility.

A (previous owner assignment) conditional priority list for object $\boldsymbol{a}, \sigma^{a}$, specifies

- an initial agent (who is the owner of the object), 
- for each possible object this initial agent is assigned, specifies a second agent who "inherits" $a$,

- for each possible object this second agent is assigned, specifies a third agent who "inherits" $a$, and so on.

A conditional priority list, $\boldsymbol{\sigma}$, is a collection of the above, one for each agent.

Define a rule as follows: Construct a graph with agents and objects as nodes. In Step 1, each object points to its initial agent, each agent points to his most preferred object, and for each cycle, assign agents the object they point to. Remove assigned agents and objects. In Step 2, each object for which 1) is still attainable given the partial allocation in Step 1, and 2) its initial agent has been assigned, points to a second agent (this may depends the assignment of the initial agent). Then, each unassigned agent points to his most preferred (attainable) object, and the process is repeated. A formal treatment of the original family of rules are in (Pápai, 2000).

The following example shows that this rule is not well-defined.

Let $E \in \mathcal{E}$ be as below. The column $R_{5,6,7}$ indicates the preference relations $R_{5}=R_{6}=$ $R_{7}$.

\begin{tabular}{ccccccc}
$R_{1}$ & $R_{2}$ & $R_{3}$ & $R_{4}$ & $R_{5,6,7}$ & $R_{8,9,10}$ & $R_{11,12,13}$ \\
\hline$\emptyset$ & $a$ & $a$ & $a$ & $a$ & $a$ & $a$ \\
$a$ & $b$ & $c$ & $d$ & $b$ & $c$ & $d$
\end{tabular}

Let the conditional priority list $\sigma$ be such that $a$ points to 1 , then for each possible object that 1 is assigned, $a$ points to 2, 3, then 4. At Step 1, 1 points to $\emptyset$ and is assigned $\emptyset$. At Step 2, a points to 2, 2 is assigned $a$. At Steps 3 and 4, 3 and 4 are assigned $a$. Let $\sigma$ be such that $b, c$, and $d$ also point at 1, 2, 3, then 4 . At Step 5, $a$ is no longer attainable given the partial allocation at Step 4 . Let $\sigma$ be such that $b$ now points to $5, c$ points to 8, and $d$ points to 11 . Then, at Step 5, agents 5, 8, and 11 are assigned object $b$, $c$, and $d$, respectively. Let $\sigma$ be such that $b, c$, and $d$ now point to 6,9 , and 10 . Then, at Step 6, 6, 9 , and 10 are assigned $b, c$, and $d$. Notice that given the partial allocation at Step $6, b, c$, and $d$ are still attainable. Let $\sigma$ be such that $b, c$, and $d$ point at 11,12 , and 13 . Then, at Step 7, agents 11, 12, and 13 are in a cycle with $b, c$, and $d$. So the rule assigns them these objects. However, $(\emptyset, a, a, a, b, b, b, c, c, c, d, d, d)$ is not feasible. In the transfer process for this allocation, there is at least one agent in $\{2,3,4\}$ who is helping 1 transfer $a$ to another agent in R2. Without loss of generality, let 2 be this agent. Then, 2 may transfer $b$ to, say, 5 in R1; and 5 may transfer $b$ to, say, 6 in R2. Since 2 is already transferring $a$ in R2, he cannot transfer $b$ to 7 .

\section{Conclusion}

We study a resource allocation problem wherein each agent may replicate and transfer an object in his possession to another agent-and there is a limited amount of time to do so. When each agent owns one object and consumes only one object, we identify a family of rules satisfying efficiency, group-strategy-proofness, withholding-proofness, and the reciprocity lower bound. If we consider the case where each agent owns bundles of objects and consumes 
bundles, then no rule satisfies the four properties. If agents live in a network, then we show that for a large class of networks, efficiency is compatible with strategy-proofness, but unfortunately, neither of the other two properties. 


\section{Appendix 1}

In Appendix 1.1, we show that $T T C-S I^{\sigma}$ satisfies non-bossiness. In Appendix 1.2, we show $T T C-S I^{\sigma}$ satisfies withholding-proofness. Let $\varphi \equiv T T C-S I^{\sigma}$.

\subsection{Non-bossiness}

Non-bossiness: For each $E=(R, \Omega) \in \mathcal{E}$, each $i \in N$, and each $R_{i}^{\prime} \in \mathcal{R}$, if $\varphi_{i}(E)=$ $\varphi_{i}\left(R_{i}^{\prime}, R_{-i}, \Omega\right)$, then

$$
\varphi(E)=\varphi\left(R_{i}^{\prime}, R_{-i}, \Omega\right)
$$

Let $E=(R, \Omega) \in \mathcal{E}$, and $E^{\prime}=\left(R_{i}^{\prime}, R_{-i}, \Omega\right) \in \mathcal{E}$ be s.t. $\varphi_{i}\left(E^{\prime}\right)=\varphi_{i}(E)$. For each $k \in\{1, \ldots, \bar{k}(E)\}$, let

- $N^{k} \equiv\{M \subseteq N$ : each $i \in M$ is a remaining agent at Step $k$ under $E\}$,

- $W^{k} \equiv\left\{w \in 2^{N}: w=\left\{w_{1}, \ldots, w_{z}\right\}\right.$, and at Step $k$ under $E, w_{z}$ points to $w_{z-1}$ points to... $w_{1}$ points to $\left.\Omega_{i}\right\}$,

- $S^{k} \equiv\left\{s \in 2^{N}: s\right.$ is a cycle $s_{1}, \ldots, s_{z}$ at Step $k$ under $E$, and there is no $t \in s$ s.t. $\left.t \in \bigcup_{g \in\{1, \ldots, k\}} \bigcup_{w \in W^{g}} w\right\}$, and

- $U^{k} \equiv\left\{s \in 2^{N} \backslash S^{k}: s\right.$ is a cycle $s_{1}, \ldots, s_{z}$ at Step $k$ under $\left.E\right\}$,

At Step $k, S^{k}$ and $U^{k}$ are the set of cycles which occur (Figure 3). In each cycle in $S^{k}$, no agent receives $\Omega_{i}$; while in $U^{k}$, some agent receives $\Omega_{i}$. At Step $k$, each $s \in W^{k}$ forms a pointing "chain" of agents for which the last agent points to $\Omega_{i}$.

Let $V$ be the Step under $E$ at which $i$ is assigned his object, and $W \in W^{V}$ be s.t. $i \in W$. Let $Q$ be the first Step under $E$ at which the cycle $W$ forms. Let $V^{\prime}$ be the Step under $E^{\prime}$ at which $i$ is assigned his object. For each $j \in N$, let $C(j, E) \subseteq N$ be the cycle $j$ is in under $E$. For each $k \in\{1, \ldots, V-1\}$, since $i$ is not assigned yet, $U^{k}=\emptyset$. For each $k \in\{V, \ldots, \bar{k}(E)\}$, either $U^{k}=\emptyset$, or there is only one cycle in $U^{k}$. Abusing notation, write $U^{k}$ as this cycle.

The proof proceeds by showing that each cycle occurring in the algorithm under $E$ occurs under $E^{\prime}$. Furthermore, for each $k \in\{1, \ldots, \bar{k}(E)\}$, each cycle occurring at Step $k$ under $E$ may not occur at an earlier round. This can be shown using the technique in Step 3 of Claim 4.

Case 1: $\quad V^{\prime}=V$. At Step 1 under $E$ and $E^{\prime}$, each agent $j \in N \backslash\{i\}$ points to the same object, the same cycles $S^{1}$ occur, and $U^{1}=\emptyset$. Since $x^{1}$ under $E$ and $E^{\prime}$ are the same, the set of attainable objects objects at Step 2 under $E$ and $E^{\prime}$ are the same. Furthermore, $N^{2}$ agents remain at Step 2 under $E$ and $E^{\prime}$.

Iterating this reasoning for Steps $2, \ldots, \bar{k}(E)$, we have $x^{2}, \ldots, x^{\bar{k}(E)}$ under $E$ and $E^{\prime}$ are the same, implying $\varphi\left(E^{\prime}\right)=\varphi(E)$. Note that $i$ may point to different objects at Step $k \in\{1, \ldots, \bar{k}(E)-1\}$, but since he is not in a cycle, this does not matter. 


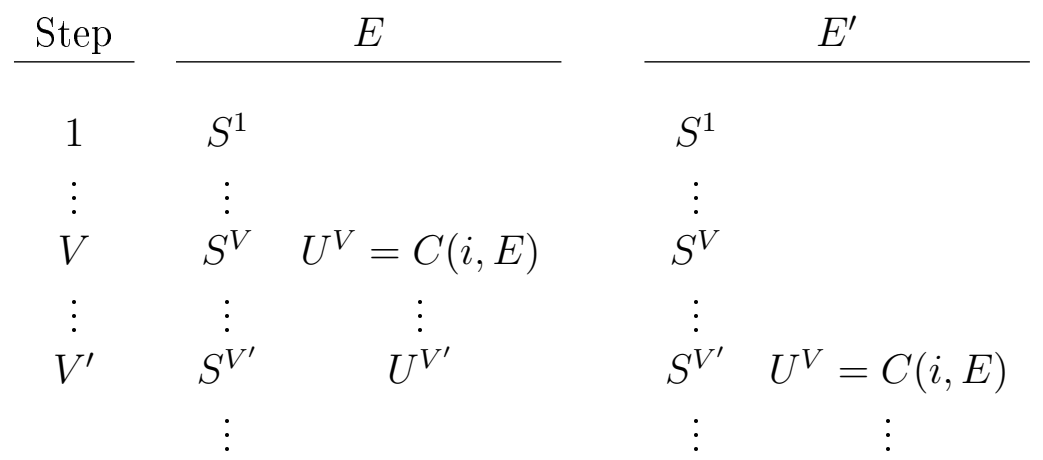

Figure 3: The schematic above shows the steps of the algorithm under $E$ and $E^{\prime}$ when $V^{\prime}>V$. Underneath the column $E, S^{1}$ and $U^{1}$ are particular sets of cycles which occur at Step 1, and similarly for each other row. Claim 1 shows cycles $S^{V}, \ldots, S^{V^{\prime}}$ occur under $E^{\prime}$; Claim 2 shows $U^{V^{\prime}}$ occurs under $E^{\prime}$.

We consider the case when $V^{\prime}>V$. If $V^{\prime}<V$, then a symmetric reasoning holds after re-labelling.

Case 2: $\quad V^{\prime}>V$. Following the reasoning of Case 1, for each $j \in \bigcup_{g \in\{1, \ldots, V\}} \bigcup_{s \in S^{g}} s$, $\varphi_{j}(E)=\varphi_{j}\left(E^{\prime}\right)$. So each agent belonging to a cycle not involving $i$ at Steps $1, \ldots, V$ receives the same assignment under $E$ and $E^{\prime}$. Since $V^{\prime}>V$, the cycle $C(i, E)$ does not occur at Step $V$ under $E^{\prime}$.

Lemma 2 If $V^{\prime}>V$, then for Step $V$ under $E$, there is no $h \in \bigcup_{k \in\{1, \ldots, V\}} \bigcup_{s \in W^{k}} s$ s.t. $h$ is the earliest-priority remaining agent.

If $V^{\prime}>V$, then at Step $V$ under $E^{\prime}, i$ does not point to $\varphi_{i}(E)$. If there is an agent that 1) points to an object that points to an agent... that points to $\Omega_{i}$ in any Step from 1 to $V$ under $E$, and 2) is the earliest-priority agent at Step $V$ under $E$, then $i$ will be assigned the object he points to at Step $V$ under $E^{\prime}$ - violating the assumption that $\varphi_{i}(E)=\varphi_{i}\left(E^{\prime}\right)$.

Proof: Suppose by contradiction, there is $k \in\{1, \ldots, V\}$, and $h \in \bigcup_{s \in W^{k}} s$ s.t. $h$ is the earliest-priority remaining agent at Step $V$ under $E$, and hence also at Step $V$ under $E^{\prime}$. Since $V^{\prime}>V$, at Step $V$ under $E^{\prime}, i$ does not point at an agent in $\bigcup_{s \in W^{k}} s$ (otherwise, he is in a cycle). Then, $i$ points to an object $a \neq \varphi_{i}(E)$ that points to $\ell \in N$, and $a R_{i}^{\prime} \varphi_{i}(E)$. If $\Omega_{\ell} \neq a$, then $\ell=h, i$ is in a cycle, and $a=\varphi_{i}\left(E^{\prime}\right) \neq \varphi_{i}(E)$-violating the assumption that $\varphi_{i}(E)=\varphi_{i}\left(E^{\prime}\right)$. If $\Omega_{\ell}=a$, then either 1) $\ell$ is in a cycle with $i$, implying $a=\varphi_{i}\left(E^{\prime}\right) \neq \varphi_{i}(E)$, or 2$) \ell$ is in a cycle, but not with $i$. First, in Step $V+1$ under $E^{\prime}$ (after $\ell$ is in a cycle), for each $s \in W^{k}$, each agent in $s$ still points to the same object as in Step $V$ under $E^{\prime}$, and hence, the same as in Step $V$ under $E$. Second, since only one agent was assigned $a$, and no agent has been assigned $\Omega_{i}, a$ is still attainable at Step $V+1$ under $E^{\prime}$. Since $h$ is the earliest-priority remaining agent at Step $V$ under $E^{\prime}$, he is so at Step $V+1$ under $E^{\prime}$. Then, $i$ points to object $a$ points to $h$, and by definition of $h \in \bigcup_{s \in W^{k}} s, h=w_{z}$ points to $w_{z-1}$ points to... points to $w_{1}$ points to $\Omega_{i}$ points to $i$. Then, $a=\varphi_{i}\left(E^{\prime}\right) \neq \varphi_{i}(E)$. 
Since $C(i, E) \in W^{V}$, Lemma 2 implies no $h \in C(i, E)$ is earliest at Step $V$ under $E$. Hence, for each $h \in C(i, E)$, at Step $V$ under $E^{\prime}, h$ points to an object in $\bigcup_{j \in C(i, E)} \Omega_{j}$. At Steps $V+1, \ldots, V^{\prime}$, each $h \in C(i, E) \backslash\{i\}$ still points to the same agent, implying $C(i, E)$ occurs at Step $V^{\prime}$ under $E^{\prime}$.

Claim 1 For each $j \in \bigcup_{k \in\left\{0, \ldots, V^{\prime}-V\right\}} \bigcup_{s \in S^{V+k}} s, \varphi_{j}\left(E^{\prime}\right)=\varphi_{j}(E)$. Furthermore, for each $k \in\left\{0, \ldots, V^{\prime}-V\right\}$, the set of cycles $S^{V+k}$ occurs at Step $V+k$ under $E$ and $E^{\prime}$, and for each $k \in\left\{1, \ldots, V^{\prime}-V-1\right\}, U^{V+k}=\emptyset$.

Proof: We have shown above that for each $j \in \bigcup_{s \in S^{V}} s, \varphi_{j}(E)=\varphi_{j}\left(E^{\prime}\right)$, and the set of cycles $S^{V}$ occurs at Step $V$ under $E$ and $E^{\prime}$.

Step 1: For each $j \in \bigcup_{k \in\left\{0, \ldots, V^{\prime}-V\right\}} \bigcup_{s \in S^{V+k}} s, \varphi_{j}\left(E^{\prime}\right)=\varphi_{j}(E)$, and for each $k \in$ $\left\{0, \ldots, V^{\prime}-V\right\}$, the set of cycles $S^{V+k}$ occurs at Step $V+k$ under $E$ and $E^{\prime}$. We proceed by induction. For each $k \in\left\{1, \ldots, V^{\prime}-V-1\right\}$, let Case $k$ be defined as below. For each $j \in \bigcup_{g \in\{0, \ldots, V+k-1\}} \bigcup_{s \in S^{V+g}}$, suppose $\varphi_{j}(E)=\varphi_{j}\left(E^{\prime}\right)$. For each $g \in\{0, \ldots, V+k-1\}$, suppose 1) $S^{g}$ occurs at Step $V+g$ under $E$ and $E^{\prime}$, and 2) $U^{V+g}=\emptyset$.

Subcase 2.k: $j \in \bigcup_{s \in S^{V+k}} s$. Then, at Step $V$ under $E$ and $E^{\prime}, j$ either points to an agent $h$ in $S^{V}, S^{V+1}, \ldots, S^{V+k}$, or $C(i, E)$. Since $U^{V+1}=\cdots=U^{V+k+-1}=\emptyset, j$ cannot point to an agent in $U^{V+1}, \ldots$, or $U^{V+k-1}$.

Subcase 2.k.1: $h$ is in $C(i, E)$. Then, $h$ still points to $i$ until Step $V^{\prime}$ under $E^{\prime}$, implying $j$ still points to $i$ at least until Step $V^{\prime}$ under $E^{\prime}$. Since $V^{\prime}>V+k$, this is impossible.

Subcase 2.k.2: $h$ is in $S^{V}, \ldots, S^{V+k-1}$. First, we show the set of attainable objects at $V+k$ under $E$ and $E^{\prime}$ are the same. Suppose by contradiction, there is an object $b$ such that $b$ is attainable at Step $V+k$ under $E^{\prime}$, but not attainable at Step $V+k$ under $E$. At Steps $V, \ldots, V+k-1$ under $E^{\prime}$, only one less cycle occurred compared to $E$ : $C(i, E)$. Hence, the occurrence of $C(i, E)$ at Step $V$ under $E$ "causes" the unattainability of $b$. If for each $\ell \in C(i, E), \varphi_{\ell}(E) \in \bigcup_{s \in C(i, E)} \Omega_{s}$, then each $c \in \bigcup_{s \in C(i, E)} \Omega_{s}$ is still attainable at Step $V+k$ under $E^{\prime}$. Hence, there are $\ell, \ell^{\prime} \in C(i, E)$ such that $\varphi_{\ell}(E) \notin \bigcup_{s \in C(i, E)} \Omega_{s}$, and $\varphi_{\ell}(E) \neq \Omega_{\ell^{\prime}}$ points to $\ell^{\prime}$. Since $\ell^{\prime} \in \bigcup_{s \in W^{V+k}} s$, by Lemma 2, this is impossible. So the set of attainable objects is the same at $V+k$ under $E$ and $E^{\prime}$.

Let $M \equiv N^{V+k} \cup C(i, E)$, and $\ell \equiv \arg \max _{g \in M} \sigma^{-1}(g)$ be the earliest-priority remaining agent at $V+k$ under $E^{\prime}$. Since $\ell \notin C(i, E), \ell=\arg \max _{g \in M \backslash C(i, E)} \sigma^{-1}(g)$, and $\ell$ is the earliest-priority remaining agent at $V+k$ under $E$. Hence, if $c$ is in the set of attainable objects given $x^{V+k-1}$ under $E$, and not in $\bigcup_{h \in C(i, E)} \Omega_{h}$, then $c$ points to the same agent $j^{c}$ at Step $V+k$ under $E$ and $E^{\prime}$.

At Step $V+k$ under $E$ and $E^{\prime}, j$ cannot point to an object in $\bigcup_{g \in C(i, E)} \Omega_{g}$, because the reasoning of Case 2.k.1 would hold. So since the set of attainable objects is the same at Step $V+k$ under $E$ and $E^{\prime}, j$ points to the same object $c$ at both Steps. As shown in the previous paragraph, $c$ points to the same agent $j^{c}$ at both Steps.

Hence, $j$ points to the same agent at $V+k$ under $E$ and $E^{\prime}$.

Subcase 2.k.3: $h$ is in $S^{V+k}$. Then, since each object and agent still points to the same agent $g$ until $g$ leaves, at Step $V+k$ under $E$ and $E^{\prime}, j$ points to the same agent. 
Hence, for each $j \in \bigcup_{s \in S^{V+k}} s$, at Step $V+k$ under $E^{\prime}$ and $E, j$ points to the same object, which in turn points to the same agent. The same set of cycles $S^{V+k}$ occurs, and $\varphi_{j}\left(E^{\prime}\right)=\varphi_{j}(E)$. Subcase $2 . k$ is complete.

Step 2: $U^{V+k}=\emptyset$. $\quad$ Let $M \equiv N^{V+k} \cup C(i, E)$. Suppose by contradiction $U^{V+k} \neq \emptyset$, then at Step $V+k$ under $E$ there are $u_{1}, \ldots, u_{z} \in U^{V+k}$ and $j \in\left\{u_{1}, \ldots, u_{z}\right\}$ s.t. $j=u_{z}$ points to $u_{z-1}$ points to... $u_{1}$ points to $\Omega_{i}$ points to $j$. This means, $j=\arg \min _{h \in M \backslash C(i, E)} \sigma^{-1}(h)$. Consider Step $V$ under $E^{\prime}$. Let $h$ be the earliest-priority remaining agent at Step $V$ under $E^{\prime}$.

If $h=i$, then since $V^{\prime} \neq V, i$ points to $a \neq \varphi_{i}(E)$. If the owner of $a$ remains, then $i$ still points to him. By finiteness of the algorithm, there is $g \in \mathbb{N}$ s.t. the owner of $a$ is assigned at $V+g$ under $E^{\prime}$, and at Step $V+g+1$ under $E^{\prime}, a$ points to $i$ and $\varphi_{i}\left(E^{\prime}\right)=a$. If the owner of $a$ does not remain, then $i$ is in a cycle with $a$, and $\varphi_{i}\left(E^{\prime}\right)=a$. This contradicts the hypothesis that $\varphi_{i}(E)=\varphi_{i}\left(E^{\prime}\right)$.

If $h \in \bigcup_{g \in\{0, \ldots, k-1\}} \bigcup_{s \in S^{V+g}} s$, then there are two cases. At Step $V+k$ under $E^{\prime}, i$ points to either an object $a \neq \varphi_{i}(E)$, or $\varphi_{i}(E)$. Let $j^{a}$ be the owner of $a$.

Case 1: At Step $V+k$ under $E, i$ points to $a \neq \varphi_{i}(E)$, and $j^{a}$ is a remaining agent. By finiteness of the algorithm, there is a $f \in\{k, \ldots, \bar{k}(E)-V\}$ such that at Step $V+f$ under $E^{\prime}, j^{a}$ is in a cycle. At Step $V+k$ under $E^{\prime}$, the facts that $M$ agents remain and $j=\arg \min _{h \in M \backslash C(i, E)} \sigma^{-1}(h)$ imply the earliest-priority remaining agent $\ell$ is such that $\ell \in C(i, E) \cup j$. Since the set of remaining agents is contained in $M, \ell$ is still the earliestpriority remaining agent at Step $V+f+1$ under $E^{\prime}$. So $a$ now points to $\ell$.

Subcase 1.1: $\ell=j$. By reasoning of Subcase 2.k.2, for each $u \in U^{V+k}$, at Step $V+k$ under $E$ and $E^{\prime}, u$ points to the same object, which in turn points to the same agent. Recall, by definition of $j$ and $U^{V+k}$, at Step $V+k$ under $E, j=u_{z}$ points to $u_{z-1}$ points to... $u_{1}$ points to $\Omega_{i}$ points to $j$. Since $i$ is a remaining agent, at Step $V+k$ under $E^{\prime}$, we have $j=u_{z}$ points to $u_{z-1}$ points to... $u_{1}$ points to $\Omega_{i}$ points to $i$ points to $a$. Each agent still points in this manner until Step $V+f+1$ under $E^{\prime}$. At Step $V+f+1$ under $E^{\prime}, j$ is the earliest-priority remaining agent, and $a$ points to $j$. Then, since $i$ points to $a$, a cycle forms and $\varphi_{i}\left(E^{\prime}\right)=a \neq \varphi_{i}(E)$, a contradiction to the hypothesis.

Subcase 1.2: $\ell \in C(i, E)$. Similarly as above, at Step $V+f+1$ under $E^{\prime}$, a points to $\ell$. Hence, there is a cycle $\left\{u_{z}, \ldots, u_{1}\right\} \subseteq C(i, E)$ such that $\ell=u_{z}$ points to $u_{z-1}$ points to... $u_{1}$ points to $\Omega_{i}$ points to $i$ points to $a$. Then, $\varphi_{i}\left(E^{\prime}\right)=a \neq \varphi_{i}(E)$, a contradiction.

Case 2: At Step $V+k$ under $E^{\prime}, i$ points to $\varphi_{i}(E)$. At Step $V$ under $E$ and $E^{\prime}$, since $x^{V-1}$ under $E$ and $E^{\prime}$ are the same, each remaining agent points to the same object. Consider agents in the cycle $C(i, E)$. Lemma 2 implies that for each $g \in C(i, E), g$ points to an object $a$ such that $a \in \bigcup_{j \in C(i, E)} \Omega_{j}$. Hence, at Steps $V, \ldots, V+k$ under $E^{\prime}$, each 
$g \in C(i, E) \backslash i$ still points to the same agent. Hence, if $i$ points to $\varphi_{i}(E)$, then $i$ is in a cycle at Step $V+k$ under $E^{\prime}$. Since $V+k<V^{\prime}$, this is impossible.

Claim 2 For each $j \in U^{V^{\prime}}, \varphi_{j}\left(E^{\prime}\right)=\varphi_{j}(E)$.

Proof: If $U^{V^{\prime}}=\emptyset$, then we are done. Let $U^{V^{\prime}} \neq \emptyset$ be a cycle.

Step 1: There is an agent in $u$ who is the earliest-priority remaining agent at Step $V^{\prime}+1$ under $E^{\prime}$. At Step $V^{\prime}$ under $E$, by definition, there is an agent in $U^{V^{\prime}}$ who is assigned $\Omega_{i}$. Since $V^{\prime}>V, i \notin N^{V^{\prime}}$ and $\Omega_{i}$ points to another agent $\ell \in U^{V^{\prime}}$. At Step $V^{\prime}+1$ under $E^{\prime}$, only one extra cycle has already occurred compared to Step $V^{\prime}$ under $E$ : $S^{V^{\prime}}$. Hence, the set of remaining agents at Step $V^{\prime}$ under $E$ contains the set of remaining agents at Step $V^{\prime}+1$ under $E^{\prime}$. Since $\ell$ is earliest at Step $V^{\prime}$ under $E$, this implies $\ell$ is earliest at Step $V^{\prime}+1$ under $E^{\prime}$.

Step 2: Each $j \in U^{V^{\prime}}$ points to the same object at Step $V^{\prime}$ under $E$ and Step $V^{\prime}+1$ under $E^{\prime}$. Let $a$ be the object $j$ points to at Step $V^{\prime}$ under $E$, and $b$ be the object $j$ points to at Step $V^{\prime}+1$ under $E^{\prime}$. Suppose by contradiction, $a \neq b$. Then at Step $V^{\prime}$ under $E$, $a$ is attainable given $x^{V^{\prime}-1}$ under $E$. At Step $V^{\prime}$ under $E$, the cycles $S^{1}, \ldots, S^{V^{\prime}-1}, C(i, E)$ have occurred; at Step $V^{\prime}+1$ under $E^{\prime}$, the cycles $S^{1}, \ldots, S^{V^{\prime}}, C(i, E)$ have occurred. Hence, at Step $V^{\prime}+1$ under $E^{\prime}$ the only extra set of cycles which occurs is $S^{V^{\prime}}$. If each cycle $s \in S^{V^{\prime}}$ is s.t. $s=u_{1}, \ldots, u_{z}$ and $u_{z}$ points to $\Omega_{u_{z-1}}$ points to $u_{z-1}$ points to $\Omega_{u_{z-2}}$ points to... $\Omega_{u_{1}}$ points to $u_{1}$ points to $\Omega_{z}$, then each object which points to an agent in $S^{V^{\prime}}$ is still attainable. Hence, there must be a cycle $s \in S^{V^{\prime}}$ and $j \in s$ s.t. $j$ does not point to an object in $\bigcup_{g \in s} \Omega_{g}$. This means some agent $\ell^{\prime} \in s$ is the earliest-priority remaining agent at Step $V^{\prime}$ under $E$. This is impossible, as we showed in Step $1, \ell \in U^{V^{\prime}}$ is the earliest-priority remaining agent.

Step 2 implies $U^{V^{\prime}}$ occurs at Step $V^{\prime}+1$ under $E^{\prime}$, and for each $j \in U^{V^{\prime}}, \varphi_{j}\left(E^{\prime}\right)=\varphi_{j}(E)$.

For each $k \in\left\{V^{\prime}+1, \ldots, \bar{k}(E)\right\}$, let

- $Q^{k} \equiv\left\{s \in 2^{N}: s\right.$ is a cycle at Step $k$ under $E$, and for each $j \in s$, there is $h \in s$ s.t. $j$ points to $\left.\Omega_{h}\right\}$, and

- $T^{k} \equiv\left\{s \in 2^{N}: s\right.$ is a cycle at Step $k$ under $E$, and there is $j \in T^{k}$ and $a \in \mathcal{O}$ s.t. $j$ points to $a$, and $\left.a \notin \bigcup_{h \in T^{k}} \Omega_{h}\right\}$.

That is, at Step $k$ under $E, Q^{k}$ is the set of cycles which only involve the endowments of the agents in the cycle, and $T^{k}$ is the cycle for which one agent points to an object whose owner has left. 
Claim 3 For each $j \in \bigcup_{s \in Q^{V^{\prime}+1} \cup T^{V^{\prime}+1}} s, \varphi_{j}\left(E^{\prime}\right)=\varphi_{j}(E)$. Furthermore, the cycles $Q^{V^{\prime}+1}$ and $T^{V^{\prime}+1}$ both occur either at $V^{\prime}+1$ under $E^{\prime}$ or $V^{\prime}+2$ under $E^{\prime}$.

Proof: If $U^{V^{\prime}}=\emptyset$, then at Step $V^{\prime}+1$ under $E$ and $E^{\prime}$, the same cycles $S^{1}, \ldots, S^{V^{\prime}}, C(i, E)$ have occurred. Hence, $x^{V^{\prime}}$ under $E$ and $E^{\prime}$ is the same, the set of remaing agents is the same, and each remaining agent at Step $V^{\prime}+1$ points to the same object under $E$ and $E^{\prime}$. So the same cycles $Q^{V^{\prime}+1}$ and $T^{V^{\prime}+1}$ occur at Step $V^{\prime}+1$ under $E^{\prime}$.

If $U^{V^{\prime}} \neq \emptyset$, then before Step $V^{\prime}$ under $E$, the cycles $S^{1}, \ldots, S^{V^{\prime}-1}, C(i, E)$ have occurred. At Step $V^{\prime}-1$ under $E^{\prime}$, only the cycles $S^{1}, \ldots, S^{V^{\prime}-1}$ have occurred.

Step 1: No agent assigned in Steps $V+1, \ldots, V^{\prime}-1$ under $E$ is assigned an object in $\bigcup_{s \in C(i, E)} \Omega_{s}$. Suppose by contradiction, there is $j \in \bigcup_{g \in\left\{1, \ldots, V^{\prime}-V-1\right\}} \bigcup_{s \in S^{V+g}} s, h \in C(i, E)$, and $k \in\left\{1, \ldots, V^{\prime}-V-1\right\}$ s.t. $j$ points to $\Omega_{h}$ at Step $V+k$ under $E$. By Claim $2, S^{V+k}$ cycles occur at Step $V+k$ under $E^{\prime}$. Since $h$ is not assigned until $V^{\prime}, \Omega_{h}$ still points to $h$ until $V^{\prime}$. Since $V^{\prime}>V+k, j$ cannot have received $\Omega_{h}$ at Step $V+k$ under $E^{\prime}$.

Step 2: The set of attainable objects at Step $V^{\prime}$ under $E$ and $E^{\prime}$ are the same. Before Step $V^{\prime}$ under $E^{\prime}$, the cycles $S^{1}, \ldots, S^{V^{\prime}-1}$ have occurred. Before Step $V^{\prime}$ under $E$, the cycles $S^{1}, \ldots, S^{V^{\prime}-1}, C(i, E)$ have occurred. Since each object $a \in \bigcup_{s \in C(i, E)} \Omega_{s}$ has only been assigned once (at Step $V$ under $E$ ), each such $a$ is attainable given $x^{V^{\prime}-1}$ under $E$. Since $C(i, E)$ comprises the only difference between $x^{V^{\prime}-1}$ under $E$ and $E^{\prime}$, the set of attainable objects at Step $V^{\prime}$ under $E$ and $E^{\prime}$ are the same.

Step 3: Each $s \in Q^{V^{\prime}+1}$ occurs at $V^{\prime}+2$ under $E$. Since the set of attainable objects given $x^{V^{\prime}-1}$ under $E$ and $E^{\prime}$ are the same, at Step $V^{\prime}$ under $E$ and $E^{\prime}$, each $j \in \bigcup_{s \in Q^{V^{\prime}+1}} s$ points to the same object. As argued before, $U^{V^{\prime}} \neq \emptyset$ implies there is an agent in $U^{V^{\prime}}$ who is the earliest-priority remaining agent at Step $V^{\prime}$ under $E$. This means, for each $s \in S^{V^{\prime}}$, each $h \in s$ points to an object $a$ s.t. there is $h^{\prime} \in s$ with $a=\Omega_{h^{\prime}}$. Hence, at Step $V^{\prime}+1$ under $E$, each $b \in \bigcup_{s \in S^{V^{\prime}}} \bigcup_{h \in s} \Omega_{h}$ is still attainable given $x^{V^{\prime}}$ under $E$. Since each $j^{\prime} \in \bigcup_{s \in Q^{V^{\prime}+1}} s$ is assigned an object in the set $\bigcup_{s \in Q^{V^{\prime}}} \bigcup_{h \in s} \Omega_{h}$, each $j^{\prime}$ points to an agent who is not in $S^{V^{\prime}}$ at Step $V^{\prime}$ under $E$. Then, at Step $V^{\prime}$ under $E$ either $j^{\prime}$ points to $\varphi_{j^{\prime}}(E)$ or an agent in $U^{V^{\prime}}$. There is at least one $h \in \bigcup_{s \in Q^{V^{\prime}+1}} s$ s.t. $h$ points to an agent in $U^{V^{\prime}}$ at Step $V^{\prime}$ under $E$, otherwise $Q^{V^{\prime}+1}$ occurs at Step $V^{\prime}$ under $E$.

Without loss of generality, let $h$ be such an agent, $a \in \bigcup_{j \in U^{V^{\prime}}} \Omega_{j}$, and $j^{a}$ be the owner of $a$. Since $j^{a}$ has not been assigned an object, $a$ has not been assigned to any agent. There is a transfer process in which each agent in $C\left(j^{a}, E\right)$ transfers to the next agent in $C\left(j^{a}, E\right)$ in the first round. Then, $a$ is still attainable given $x^{V^{\prime}}$ under $E: j^{a}$ transfers to another agent in the second round. Hence, if $h$ points to $a$ at Step $V^{\prime}$ under $E$, then $h$ still points to $a$ at Step $V^{\prime}+1$ under $E$. By definition of $Q^{V^{\prime}}+1$, there is $h^{\prime} \in N^{V^{\prime}+1}$ s.t. $h$ is assigned $\Omega_{h^{\prime}}$. Since $j^{a} \notin N^{V^{\prime}+1}$, this is a contradiction. Hence, at Step $V^{\prime}$ under $E, h$ points to an object $a \notin \bigcup_{j \in U^{V^{\prime}}} \Omega_{j}$, and $a$ points to an agent in $U^{V^{\prime}}$. Recall there is $u \in U^{V^{\prime}}$ s.t. $u$ is the earliest-priority remaining agent at Step $V^{\prime}$ under $E$, and by definition of $U^{V^{\prime}}, \Omega_{i}$ is assigned 
to some agent. In $C(u, E)$, since $\Omega_{i}$ points to $u$, and $\Omega_{i}$ is the only object which does not point to its owner, at Step $V^{\prime}$ under $E, h$ must point to $\Omega_{i}{ }^{22}$

Since no agent in $\bigcup_{s \in S^{V^{\prime}}} s$ points to $\Omega_{i}$ at Step $V^{\prime}$ under $E$ and $E^{\prime}, \Omega_{i}$ is attainable given $x^{V^{\prime}}$ under $E^{\prime}$. As shown above, at Step $V^{\prime}$ under $E, h$ points to $\Omega_{i}$. Since the set of attainable objects at Step $V^{\prime}+1$ under $E^{\prime}$ is the same as the set of attainable objects at Step $V^{\prime}$ under $E$, at Step $V^{\prime}+1$ under $E^{\prime}, h$ points to $\Omega_{i}$. Since $U^{V^{\prime}} \neq \emptyset$, at Step $V^{\prime}+1$ under $E^{\prime}, \Omega_{i}$ points to an agent in $U^{V^{\prime}}$, and $h$ is not assigned.

At Step $V^{\prime}$ under $E$ and $E^{\prime}$, each $j \in\left\{\bigcup_{s \in Q^{V^{\prime}+1}} s\right\} \backslash h$ points to $\varphi_{j}(E)$. Furthermore, at Step $V^{\prime}$ under $E^{\prime}$, there is $j \in\left\{\bigcup_{s \in Q^{V^{\prime}+1}} s\right\} \backslash h$ which points to $h$. Since $h$ is not assigned at Step $V^{\prime}+1$ under $E^{\prime}$, each agent in $\bigcup_{s \in Q^{V^{\prime}+1}} s$ is a remaining agent at Step $V^{\prime}+2$ under $E^{\prime}$.

At Step $V^{\prime}+2$ under $E^{\prime}$, the same set of cycles $S^{1}, \ldots, S^{V^{\prime}}, C(i, E), U^{V^{\prime}}$ have occurred under $E$ and $E^{\prime}$, implying $x^{V^{\prime}}$ under $E$ and $x^{V^{\prime}+1}$ under $E^{\prime}$ are the same. Hence, at Step $V^{\prime}+2$ under $E^{\prime}$, each $j \in \bigcup_{s \in Q^{V^{\prime}+1}} s$ points to the same object $a$ which $j$ points to at Step $V^{\prime}+1$ under $E$. Then cycles $Q^{V^{\prime}+1}$ occurs at Step $V^{\prime}+2$ under $E^{\prime}$, and for each $j \in \bigcup_{s \in Q^{V^{\prime}+1}} s, \varphi_{j}\left(E^{\prime}\right)=\varphi_{j}(E)$.

Step 4: $T^{V^{\prime}+1}$ occurs at Step $V^{\prime}+2$ under $E^{\prime}$. Since the set of attainable objects given $x^{V^{\prime}-1}$ under $E$ and $E^{\prime}$ are the same, at Step $V^{\prime}$ under $E$ and $E^{\prime}$, each $j \in T^{V^{\prime}+1}$ points to the same object.

Without loss of generality, let $h \in T^{V^{\prime}+1}$ be such that at Step $V^{\prime}$ under $E, h$ points to an agent in $S^{V^{\prime}}$ or $U^{V^{\prime}}$. Such an $h$ exists, otherwise $T^{V^{\prime}+1}$ may occur at a step under $E$ before $T^{V^{\prime}+1}$. Since the set of attainable objects given $x^{V^{\prime}-1}$ under $E$ and $E^{\prime}$ is the same, $h$ points to the same object at Step $V^{\prime}$ under $E$ and $E^{\prime}$. The only difference from Step 4 is that $h \in T^{V^{\prime}+1}$ can point to an agent in $S^{V^{\prime}}$.

Case 1: At Step $V^{\prime}$ under $E, h$ points to an agent in $S^{V^{\prime}}$. Then $S^{V^{\prime}}$ occurs at Step $V^{\prime}$ under $E^{\prime}$, and $h$ is not assigned an object. Since at $V^{\prime}+1$ under $E^{\prime}, U^{V^{\prime}}$ is the only cycle, $h$ is not assigned at this step either. At Step $V^{\prime}+2$ under $E^{\prime}$, the same set of cycles $S^{1}, \ldots, S^{V^{\prime}}, U^{V^{\prime}}, C(i, E)$ have occurred as in Step $V^{\prime}+1$ under $E$. Hence, the set of attainable objects given $x^{V^{\prime}+1}$ under $E^{\prime}$ and $x^{V^{\prime}}$ under $E$ is the same. So each $j \in T^{V^{\prime}+1}$ points to the same at Step $V^{\prime}+2$ under $E^{\prime}$ and Step $V^{\prime}+1$ under $E-T^{V^{\prime}+1}$ occurs at Step $V^{\prime}+2$ under $E^{\prime}$.

Case 2: At Step $V^{\prime}$ under $E, h$ points to an agent in $U^{V^{\prime}}$. Then since $U^{V^{\prime}}$ does not occur until Step $V^{\prime}+1$ under $E^{\prime}, h$ is a remaining agent at Step $V^{\prime}+2$ under $E^{\prime}$.

As before $x^{V^{\prime}}$ under $E$ and $x^{V^{\prime}+1}$ under $E^{\prime}$ are the same, each agent $j \in T^{V^{\prime}+1}$ points to the same object at Step $V^{\prime}+2$ under $E^{\prime}$ and $V^{\prime}+1$ under $E$. Then, $T^{V^{\prime}+1}$ occurs at Step $V^{\prime}+2$ under $E^{\prime}$, and for each $j \in T^{V^{\prime}+1}, \varphi_{j}\left(E^{\prime}\right)=\varphi_{j}(E)$.

\footnotetext{
${ }^{22}$ This further implies at Step $V^{\prime}+1, \Omega_{i}$ is not attainable. The only time this is possible is the following situation. Let 1 be the owner of $a=\varphi_{i}(E)$. Then 1 transfers $a$ to $2,3, i ; 2$ transfers $b$ to $4,5,6$; and 3 transfers $c$ to 7,8,9. Since $i$ must help 1 transfer $a$, only two agents may receive object $\Omega_{i}$.
} 


\begin{tabular}{|c|c|c|c|c|}
\hline Step & \multicolumn{2}{|r|}{$E$} & \multicolumn{2}{|r|}{$E^{\prime}$} \\
\hline 1 & $S^{1}$ & & $S^{1}$ & \\
\hline$\vdots$ & $\vdots$ & & $\vdots$ & \\
\hline$V$ & $S^{V}$ & $U^{V}=C(i, E)$ & $S^{V}$ & \\
\hline$\vdots$ & $\vdots$ & $\vdots$ & $\vdots$ & \\
\hline$V^{\prime}$ & $S^{V^{\prime}}$ & $U^{V^{\prime}}$ & $S^{V^{\prime}}$ & $U^{V}=C(i, E)$ \\
\hline$V^{\prime}+1$ & $Q^{V^{\prime}+1}$ & $T^{V^{\prime}+1}$ & & $U^{V^{\prime}}$ \\
\hline$V^{\prime}+2$ & & & $Q^{V^{\prime}+1}$ & $T^{V^{\prime}+1}$ \\
\hline
\end{tabular}

Figure 4: Cycles $Q^{V^{\prime}+1}$ and $T^{V^{\prime}+1}$ occur at Step $V^{\prime}+1$ under $E$, but under $E^{\prime}$, when $U^{V^{\prime}} \neq \emptyset$, they occur at Step $V^{\prime}+2$.

Claim 4 For each $k \in\left\{1 \ldots, \bar{k}(E)-V^{\prime}\right\}$, and each $j \in \bigcup_{s \in Q^{V^{\prime}+k} \cup T^{V^{\prime}+1}} s, \varphi_{j}\left(E^{\prime}\right)=$ $\varphi_{j}(E)$.

Proof: We have proved the statement for $k=1$ above, and proceed by induction. Let the statement be true for $k=2, \ldots, k-1$. If $Q^{V^{\prime}+k}=T^{V^{\prime}+k}=\emptyset$, then the algorithm has terminated and we are done. If $Q^{V^{\prime}+k}=\emptyset$ and $T^{V^{\prime}+k} \neq \emptyset$, then proceed to Step 4 of the proof.

Let $Q^{V^{\prime}+k} \neq \emptyset$.

Step 1: $T^{V^{\prime}+k-1} \neq \emptyset$. Suppose by contradiction, $T^{V^{\prime}+k-1}=\emptyset$. By definition of $Q^{V^{\prime}+k-1}$, for each $s \in Q^{V^{\prime}+k-1}$, and each $h \in s, \varphi_{h}(E) \in \bigcup_{t \in C(h, E)} \Omega_{t}$. Let $h^{a}$ be the agent assigned $a$ at Step $V^{\prime}+k-1$ under $E$. For each $a \in \bigcup_{t \in C(h, E)} \Omega_{t}$, there is a transfer process and $h^{\prime} \in N \backslash s$ which assigns to $h^{\prime}$ object $a$ : in R1, the owner of $a$ replicates and transfers to $h^{a}$, and in R2, to $h^{\prime}$. Since each object $b$ attainable given $x^{V^{\prime}+k-2}$ under $E$ which is not in $\bigcup_{t \in C(h, E)} \Omega_{t}$ is not assigned to a new agent, $b$ is still attainable given $x^{V^{\prime}+k-1}$ under $E$. Hence, each object which is attainable given $x^{V^{\prime}+k-2}$ under $E$ is attainable given $x^{V^{\prime}+k-1}$ under $E$. At Step $V^{\prime}+k$ under $E$, each agent in $N^{V^{\prime}+k}$ points to the same object as the previous Step, but since $s^{\prime} \in Q^{V^{\prime}+k}$ is a cycle, $s^{\prime}$ must have happened at Step $V^{\prime}+k-1$-violating the definition of the rule.

Step 2: Each $j \in \bigcup_{s \in Q^{V^{\prime}+k}} s, j$ is a remaining agent at Step $V^{\prime}+k$ under $E^{\prime}$, and each cycle $s \in Q^{V^{\prime}+k}$ eventually occurs under $E^{\prime} . \quad$ For each $s \in Q^{V^{\prime}+k}$, there is $h \in s$ s.t. at Steps $1, \ldots, V^{\prime}+k-1$ under $E, h$ points to an agent $g \notin C(h, E)$; otherwise, the cycle $C(h, E)$ forms before Step $V^{\prime}+k$ under $E$. At Steps $1, \ldots, V-1$ under $E$ and $E^{\prime}$, the algorithm is the same, so $h \in N^{V}$ implies $h$ is a remaining agent at Step $V$ under $E^{\prime}$. Furthermore, since $x^{V-1}$ under $E$ and $E^{\prime}$ is the same, $h$ points to the same object at Step $V$ under $E$ and $E^{\prime}$. Let $G \equiv\left\{C(i, E), S^{V}, \ldots, S^{V^{\prime}}, U^{V^{\prime}}, Q^{V^{\prime}+1}, \ldots, Q^{V^{\prime}+k-1}, T^{V^{\prime}+1}, \ldots, T^{V^{\prime}+k-1}\right\}$ be the set of all cycles which occur during Steps $V, \ldots, V^{\prime}+k-1$ under $E$.

For each $t \in\left\{0, \ldots, V^{\prime}-V\right\}$, and each Step $V+t$ under $E, h$ points to an agent in $\bigcup_{g \in G} \bigcup_{s \in g} s$. Case 1: At Step $V$ under $E, h$ points to an agent in $C(i, E)$. Then, 
at Steps $V, \ldots, V^{\prime}$ under $E^{\prime}, h$ points to the same agent. Case 2: There are sequences $g_{1}, \ldots, g_{z} \in\left\{0, \ldots, V^{\prime}-V\right\}$, and $f_{1}, \ldots, f_{z} \in N$ s.t.

- $g_{1}<\cdots<g_{z}$

- $g_{z}=V^{\prime}-V$,

- for each $m \in\{1, \ldots, z\}, f_{m} \in \bigcup_{s \in S^{g m} \cup U^{V^{\prime}}} s$,

- $h$ points to $f_{1}$ at Steps $V, \ldots, V+g_{1}$ under $E$, and

- for each $m \in\{2, \ldots, z\}, h$ points to $f_{m}$ at Steps $V+g_{y-1}+1, \ldots, V+g_{y}$ under $E$.

Since $h$ points to the same object at Step $V$ under $E$ and $E^{\prime}$, and the set of remaining agents at Step $V$ under $E$ and $E^{\prime}$ is the same, $h$ points to $f_{1}$ at Step $V$ under $E^{\prime}$. We will show $h$ is a remaining agent at Step $V^{\prime}$ under $E^{\prime}$.

If $f_{1} \in C(i, E)$, then at Steps $V, \ldots, V^{\prime}$ under $E^{\prime}, h$ points to $f_{1}$.

If there is $m \in\{1, \ldots, z\}$ s.t. at Steps $V+g_{m-1}+1, \ldots, V+g_{m}$ under $E, f_{m}$ is the earliest-priority remaining agent and $h$ points to $a \in \bigcup_{s \in C(i, E)} \Omega_{s}$ (which points to $f_{m}$ ), then $h$ points to an agent in $C(i, E)$ at Step $V^{\prime}$ under $E^{\prime}$.

Let $A \equiv \bigcup_{k \in\{1, \ldots, V-1\}} \bigcup_{s \in S^{k}} s$. If there is $m \in\{1, \ldots, z\}$ s.t. at Steps $V+g_{m-1}, \ldots, V+g_{m}$ under $E, f_{m}$ is the earliest- priority remaining agent, $h$ points to $a \in \bigcup_{s \in C(i, E) \cup A} \Omega_{s}$, and there is $j \in C(i, E)$ s.t. $j$ is the earliest-priority remaining agent in the set $N^{V+g_{m-1}+1} \cup j$, then at Steps $V, \ldots, V+g_{m-1}-1$ under $E^{\prime}, h$ points to the same sequence of agents $f_{1}, \ldots, f_{m-1}$, and at Steps $V+g_{m-1}, \ldots, V^{\prime}$ under $E^{\prime}, h$ points to $j$.

If the previous cases do not hold, then $h$ points to the same sequence of agents $f_{1}, \ldots, f_{z}$ at Steps $V, \ldots, V^{\prime}$ under $E$ and $E^{\prime}$. In all cases, $h$ is a remaining agent at Step $V^{\prime}$ under $E^{\prime}$.

Next, we show $h$ is a remaining agent at Step $V^{\prime}+k$ under $E^{\prime}$.

If $U^{V^{\prime}}=\emptyset$, then the same set of cycles $Q^{V^{\prime}}, T^{V^{\prime}}, Q^{V^{\prime}+1}, T^{V^{\prime}+1}, \ldots, Q^{V^{\prime}+k-1}, T^{V^{\prime}+k-1}$ occur at Steps $V^{\prime}, \ldots, V^{\prime}+k-1$ under $E$ and $E^{\prime}$. Hence, we can construct a sequence as above, and $h$ points to the same sequence of agents at Steps $V^{\prime}, \ldots, V^{\prime}+k-1$. So $h$ is a remaining agent at Step $V^{\prime}+k$ under $E$.

If $U^{V^{\prime}} \neq \emptyset$, then as shown previously, the set of attainable objects given $x^{V^{\prime}-1}$ under $E$ and $x^{V^{\prime}-1}$ under $E^{\prime}$ is the same and $h$ points to the same object at Step $V^{\prime}$ under $E$ and $E^{\prime}$.

Let $u \in U^{V^{\prime}}$, and $j \in u$ be s.t. $j$ is the earliest-priority remaining agent. This means $j$ is the earliest-priority remaining agent at Step $V^{\prime}$ under $E: N^{V} \backslash\left\{C(i, E) \cup\left\{\bigcup_{s \in S^{V}, \ldots, S^{V^{\prime}-1}} s\right\}\right\}$. At Step $V^{\prime}$ under $E^{\prime}$, the set of remaining agents is $N^{V} \backslash\left\{\bigcup_{s \in S^{V}, \ldots, S^{V^{\prime}-1}} s\right\}$. Hence, at Step $V^{\prime}$ under $E^{\prime}$, either $j$ is the earliest-priority remaining agent, or there is $j^{\prime} \in C(i, E)$ s.t. $j^{\prime}$ is earlier w.r.t $\sigma$.

At Step $V^{\prime}$ under $E, h$ either points to an agent in $S^{V^{\prime}}$ or $U^{V^{\prime}}$. If $h$ points to an agent in $S^{V^{\prime}}$, then he is a remaining agent at Step $V^{\prime}$ under $E^{\prime}$. If $h$ points to an object $a$ which points to an agent in $U^{V^{\prime}}$, then at Step $V^{\prime}$ under $E^{\prime}$, there is either $j \in \bigcup_{s \in U^{V^{\prime}}} s$ or $j^{\prime} \in C(i, E)$ s.t. $j$ or $j^{\prime}$ is the earliest-priority remaining agent. If $a \notin \bigcup_{s \in C(i, E)} \Omega_{s}$ and $j$ is the earliest, then $U^{V^{\prime}}$ occurs at Step $V^{\prime}+1$ under $E^{\prime}$; so $h$ still points to $j$ at Step $V^{\prime}$ and $V^{\prime}+1$ under $E^{\prime}$. If $a \in \bigcup_{s \in C(i, E)} \Omega_{s}$ or $j^{\prime}$ is the earliest, then $h$ points to $j^{\prime}$ at Step $V^{\prime}$ under $E^{\prime}$, and since $C(i, E)$ occurs at Step $V^{\prime}$ under $E^{\prime}, h$ is not assigned an object. Furthermore, at $V^{\prime}+1$ 
under $E^{\prime}$, in either case, $h$ points to an agent in $U^{V^{\prime}}$. Since $U^{V^{\prime}}$ occurs at Step $V^{\prime}+1$ under $E^{\prime}, h$ is not assigned at $V^{\prime}+1$ under $E^{\prime}$.

If $U^{V^{\prime}}=\emptyset$, then let $D\left(V^{\prime}+1\right)=V^{\prime}+1$. If $U^{V^{\prime}} \neq \emptyset$, then let $D\left(V^{\prime}+1\right)=V^{\prime}+2$.

For each $z \in\{2, \ldots, k-1\}$, let $D\left(V^{\prime}+z\right)$ be the step at which $Q^{V^{\prime}+z}$ and $T^{V^{\prime}+z}$ cycles occurs under $E^{\prime}$ (by assumption this is true). Let $z \in\{1, \ldots, k-1\}$. Before Step $D\left(V^{\prime}+z\right)$ under $E^{\prime}$ and Step $V^{\prime}+z$ under $E$, the same cycles $H \equiv\left\{S^{1}, \ldots, S^{V^{\prime}}, C(i, E), U^{V^{\prime}}, Q^{V^{\prime}+1}\right.$, $\left.\ldots, Q^{V^{\prime}+z-1}, T^{V^{\prime}}, \ldots, T^{V^{\prime}+z-1}\right\}$ have occurred. This implies $x^{D\left(V^{\prime}+z-1\right)}$ under $E^{\prime}$ and $x^{V^{\prime}+z-1}$ under $E$ are the same, implying at Step $D\left(V^{\prime}+z\right)$ under $E^{\prime}$ and Step $V^{\prime}+z$ under $E, h$ points to the same agent. Since at Step $V^{\prime}+z$ under $E, h$ is not in a cycle, at Step $D\left(V^{\prime}+z\right)$ under $E^{\prime}, h$ is not in a cycle either.

Hence, $h$ is a remaining agent at Step $D\left(V^{\prime}+k-1\right)+1$ under $E^{\prime}$, and $h$ points to the same agent as in Step $V^{\prime}+k$ under $E$. Under analagous reasoning as for $h$, for each $j \in \bigcup_{s \in Q^{V^{\prime}+k}} s, j$ is a remaining agent at Step $D\left(V^{\prime}+k-1\right)+1$ under $E^{\prime}$, and $j$ points to the same agent as in Step $V^{\prime}+k$ under $E$. Since $Q^{V^{\prime}+k}$ cycles occur at Step $V^{\prime}+k$ under $E, Q^{V^{\prime}+k}$ cycles occur at Step $D\left(V^{\prime}+k-1\right)+1$ under $E^{\prime}$.

Step 3: $T^{V^{\prime}+k}$ occurs at Step $D\left(V^{\prime}+k-1\right)+1$ under $E^{\prime} . \quad$ If $T^{V^{\prime}+k}=\emptyset$, then we are done. Let $T^{V^{\prime}+k} \neq \emptyset$. As before, there is $h \in \bigcup_{s \in T^{V^{\prime}+k}} s$ s.t. at Steps $1, \ldots, V^{\prime}+k-1$ under $E, h$ does not point to any agent in $C(h, E)$. The same reasoning holds as if $h \in \bigcup_{s \in Q^{V^{\prime}+k}} s$. This implies (as shown above) each $j \in \bigcup_{s \in T^{V^{\prime}+k}} s$ is a remaining agent at Step $D\left(V^{\prime}+k-1\right)+1$ under $E^{\prime}$. The same cycles $H$ have occurred before Step $D\left(V^{\prime}+k-1\right)+1$ under $E^{\prime}$ and $V^{\prime}+k$ under $E$. This means the set of remaining agents at these two steps are the same, implying the earliest-priority remaining agent is the same. Also as above, each $j \in \bigcup_{s \in T^{V^{\prime}+k}} s$ points to the same object at Step $D\left(V^{\prime}+k-1\right)+1$ under $E^{\prime}$ and Step $V^{\prime}+k$ under $E$. The two previous statements imply cycles $T^{V^{\prime}+k}$ occur at Step $D\left(V^{\prime}+k-1\right)+1$ under $E^{\prime}$.

\subsection{Withholding-proofness}

Let $Q$ be the first step under $E$ that there is $j \in N$ which points to $\Omega_{i}, E^{\prime} \equiv\left(R, \Omega_{i}=\emptyset, \Omega_{-i}\right)$, and $V^{\prime}$ be the step under $E^{\prime}$ at which $i$ is assigned an object.

Case 1: $Q>V$. Steps $1, \ldots, V$ under $E$ and $E^{\prime}$ are the same, so $\varphi_{i}(E)=\varphi_{i}\left(E^{\prime}\right)$.

Case 2: $Q=V$. Steps $1, \ldots, V-1$ under $E$ and $E^{\prime}$ are the same, so at Step $V$ under $E$ and $E^{\prime}$, the set of attainable objects are the same, and $i$ points to the same object. If $V^{\prime}=V$, then $\varphi_{i}(E)=\varphi_{i}\left(E^{\prime}\right)$. Let $V^{\prime}>V$. For each $k \in\left\{V-1, \ldots, V^{\prime}-1\right\}$, and each $j \in N$ s.t. $x_{j}^{V-1}$ under $E$ is not $\emptyset, x_{j}^{k}$ under $E^{\prime}$ is the same as $x_{j}^{V-1}$ under $E$. By Lemma 1 , the set of attainable objects given $x^{V^{\prime}-1}$ under $E$ is contained in the set of attainable objects given $x^{V-1}$ under $E$. Hence, $i$ points to his most preferred object in a smaller set at Step $V^{\prime}$ under $E^{\prime}$, implying $\varphi_{i}(E) R_{i} \varphi_{i}\left(E^{\prime}\right)$.

Case 3: $Q<V$.

Step 1: If $i$ is assigned an object at Step $V \leq V^{\prime}$, then $\varphi_{i}(E) R_{i} \varphi_{i}\left(E^{\prime}\right)$. For each $k \in\{0,1, \ldots, V-1-Q\}$, let

- $J^{k} \equiv\{j \in N$ : at Step $Q+k$ under $E, j$ points to $i\}$, 
- $S^{k} \equiv\left\{s \subseteq 2^{N}: s\right.$ is a cycle $s_{1}, \ldots, s_{z}$ at Step $Q+k$ under $\left.E\right\}$,

- $T^{k} \equiv\left\{t \subseteq 2^{N \backslash\left\{J^{0} \cup \cdots \cup J^{k}\right\}}: t\right.$ is a cycle $t_{1}, \ldots, t_{z}$ at Step $Q+k$ under $\left.E^{\prime}\right\}$, and

- $U^{k} \equiv\left\{t \subseteq 2^{N}: t\right.$ is a cycle at Step $Q+k$ under $E^{\prime}$, and there is $j \in\left\{J^{0} \cup \cdots \cup J^{k}\right\}$ s.t. $j \in t\}$.

\begin{tabular}{cccccc} 
Step & & $E$ & & \multicolumn{2}{c}{$E^{\prime}$} \\
\cline { 1 - 1 } \cline { 5 - 6 }$Q$ & & $S^{0}$ & & $T^{0}$ & $U^{0}$ \\
$Q+1$ & & $S^{1}$ & & $T^{1}$ & $U^{1}$ \\
$\vdots$ & & $\vdots$ & & $\vdots$ & $\vdots$ \\
$V^{\prime}$ & & $S^{V^{\prime}-Q}$ & & $T^{V^{\prime}-Q}$ & $U^{V^{\prime}-Q}$
\end{tabular}

Figure 5: At Step $Q+k$ under $E$ the set of all cycles is $S^{k}$. At Step $Q+k$ under $E^{\prime}$, the set of cycles divided into two groups: $T^{k}$ is group "unaffected" by the fact that $i$ has withheld, and $U^{k}$ is the group "affected" by the fact that $i$ has withheld (because there is some agent who pointed at $\left.\Omega_{i}\right)$.

Claim For each $k \in\{0, \ldots, V-1-Q\}, S^{k}=T^{k}$.

Proof: Steps $1, \ldots, Q-1$ under $E$ and $E^{\prime}$ are the same, implying the set of attainable objects at Step $Q$ under $E$ and $E^{\prime}$ are the same, and the earliest-priority remaining agent at Step $Q$ under $E$ and $E^{\prime}$ is the same. Hence, each $j \in N^{Q} \backslash J^{0}$ points to the same agent $j^{\prime} \in N^{Q} \backslash J^{0}$, and $S^{0}=T^{0}$.

By definition, for each $u \in U^{0}$, there is $\ell \in J^{0}$ s.t. $\ell \in u$. At Step $Q+1$ under $E, \ell$ points to $\Omega_{i}$, implying that the earliest step $\ell$ can be in a cycle is $V$. Hence, $\ell \notin \bigcup_{s \in S^{1}} s$. Let $j \in \bigcup_{s \in S^{1}} s$. At Step $Q+1$ under $E$, if $j$ points to an agent in $\bigcup_{s \in U^{0}} s$, then he will remain until at least Step $V$ under $E$ - a contradiction. So $\left\{\bigcup_{s \in S^{1}} s\right\} \bigcap\left\{\bigcup_{s \in U^{0}} s\right\}=\emptyset$.

Next we show for each $s \in S^{1}$, and each $j \in s, j$ points to the same object at Steps $Q+1$ under $E$ and $E^{\prime}$. The only difference between $x^{Q}$ under $E$ and $E^{\prime}$ is the assignment of agents in the set of cycles $U^{0}$. At Step $Q$ under $E^{\prime}$, if each $h \in \bigcup_{s \in U^{0}} s$ points to object in $\bigcup_{s \in U^{0}} \bigcup_{g \in s} \Omega_{g}$, then the set of attainable objects given $x^{Q}$ under $E$ and $E^{\prime}$ are the same and $j$ points to the same object at Step $Q+1$ under $E$ and $E^{\prime}$-implying $S^{1}=T^{1}$. If instead, there is $h \in \bigcup_{s \in U^{0}} s$ s.t. $h$ is assigned $a \notin \bigcup_{s \in U^{0}} \bigcup_{g \in s} \Omega_{g}$, then there is $h^{\prime} \in \bigcup_{s \in U^{0}} s$ s.t. $h^{\prime}$ is the earliest-priority remaining agent in the set $N^{Q}$, and consequently the set $N^{Q} \backslash \bigcup_{s \in S^{0}} s$. This implies $h^{\prime}$ is the earliest-priority remaing agent at Step $Q+1$ under $E$. Since $\left\{\bigcup_{s \in S^{1}} s\right\} \bigcap\left\{\bigcup_{s \in U^{0}} s\right\}=\emptyset$, for each $s \in S^{1}$, and each $j \in s$, at Step $Q+1$ under $E$, the only object which points to $j$ is $\Omega_{j}$. Hence, at Step $Q+1$ under $E$, each $j \in \bigcup_{s \in S^{1}} s$ points to object $a^{j} \in \bigcup_{g \in s} \Omega_{g}$. Since the set of attainable objects at Step $Q+1$ under $E$ contains the set of attainable objects at Step $Q+1$ under $E^{\prime}$, the fact that for each $j \in \bigcup_{s \in S^{1}} s$, at Step $Q+1$ under $E, j$ points to $a^{j}$ implies at Step $Q+1$ under $E^{\prime}, j$ points to $a^{j}$. This implies each $s \in S^{1}$ occurs at Step $Q+1$ under $E^{\prime}$, and $S^{1}=T^{1}$.

The rest of the proof is completed by iteration of the previous reasoning for $S^{2}, \ldots, S^{V-1}$. The following is a demonstration for $S^{2}=T^{2}$. 
Let $j \in \bigcup_{s \in S^{2}} s$, and $a$ be the object $j$ points to at Step $Q+2$ under $E$, and $b$ be the object $j$ points to at Step $Q+2$ under $E^{\prime}$. The only difference between $x^{Q+1}$ under $E$ and $E^{\prime}$ is the assignment of agents in the set of cycles $U^{0}$ and $U^{1}$.

If for each $m \in\{0,1\}$, each $s \in U^{m}$, and each $h \in s$, at Step $Q+m$ under $E^{\prime}, h$ points to object in $\bigcup_{g \in s} \Omega_{s}$, then the set of attainable objects given $x^{Q+1}$ under $E$ and $E^{\prime}$ are the same, and $a=b$.

If there is $h \in \bigcup_{s \in U^{0}} s$ s.t. $h$ is assigned $d \notin \bigcup_{s \in U^{0}} \bigcup_{g \in s} \Omega_{g}$, then there is $h^{\prime} \in \bigcup_{s \in U^{0}} s$ s.t. $h^{\prime}$ is the earliest-priority remaining agent in the set $N^{Q}$. Since $h^{\prime}$ remains at least until Step $V$ under $E, h^{\prime}$ is still the earliest-priority remaining agent at Steps $Q+1$ and $Q+2$ under $E$. Similarly as before, $\left\{\bigcup_{s \in S^{2}} s\right\} \bigcap\left\{\bigcup_{s \in U^{0}} s\right\}=\emptyset$. Hence, for each $s \in S^{2}$, and each $j \in s$, at Step $Q+2$ under $E$, the only object which points to $j$ is $\Omega_{j}$. This implies that at Step $Q+2$ under $E$, for each $s \in S^{2}$ and each $j \in s, j$ points to an object $a^{j} \in \bigcup_{g \in s} \Omega_{g}$. Since the set of attainable objects given $x^{Q+1}$ under $E$ contains the set of attainable objects given $x^{Q+1}$ under $E^{\prime}$, but each $a^{j} \in \bigcup_{g \in s} \Omega_{g}$ is still attainable given $x^{Q+1}$ under $E^{\prime}$, for each $s \in S^{2}$, and each $j \in s, j$ points to $a^{j}$ at Step $Q+2$ under $E^{\prime}$. Meaning $a=b$.

If there is $h \in \bigcup_{s \in U^{1}} s$ s.t. $h$ is assigned $d \notin \bigcup_{s \in U^{1}} \bigcup_{j \in s} \Omega_{j}$, then there is $h^{\prime} \in \bigcup_{s \in U^{0}} s$ s.t. $h^{\prime}$ is the earliest-priority remaining agent in the set $N^{Q+1}$. The reasoning of above holds.

For each $i \in N$ s.t. $x_{i}^{V-1}$ under $E$ is not $\emptyset$, either

1. $i$ was assigned in $\{1, \ldots, Q-1\}$, implying $x_{i}^{V-1}$ under $E^{\prime}$ and $E$ are the same; or,

2. $i \in \bigcup_{k \in\{0, \ldots, V-1-Q\}} \bigcup_{s \in S^{k}} s=\bigcup_{k \in\{0, \ldots, V-1-Q\}} \bigcup_{t \in T^{k}} t$, implying $x_{i}^{V-1}$ under $E^{\prime}$ and $E$ are the same.

By Lemma 1, the set of attainable objects given $x^{V-1}$ under $E$ contains the set of attainable objects given $x^{V-1}$ under $E^{\prime}$. Since $i$ points to his most preferred object from the former set, $\varphi_{i}(E) R_{i} \varphi_{i}\left(E^{\prime}\right)$. We have completed Step 1 .

Now we need to show that $\varphi_{i}(E) R_{i} \varphi_{i}\left(E^{\prime}\right)$ when $V>V^{\prime}$. For intuition, we give the proof of the case when $V^{\prime}=Q+1$.

Claim If $V^{\prime}=Q+1$, then $\varphi_{i}(E) R_{i} \varphi_{i}\left(E^{\prime}\right)$.

Proof: If $V \leq V^{\prime}$, then by Step 1, we are done. Let $V>V^{\prime}$. Since $U^{0}$ is the only additional assignment made in $x^{Q}$ under $E^{\prime}$ compared to $x^{Q}$ under $E$, by Lemma 1 , the set of attainable objects at Step $Q+1$ under $E$ contains the set of attainable objects at Step $Q+1$ under $E^{\prime}$. Let $a$ be the object $i$ points to at Step $Q+1$ under $E$.

Case 1: $a \neq \varphi_{i}\left(E^{\prime}\right)$. Since $U^{0}$ is the only additional set of cycles occuring before Step $Q+1$ under $E^{\prime}$ compared to Step $Q+1$ under $E, U^{0}$ must have "caused" the unattainability of $a$ after Step $Q$ under $E^{\prime}$. The two ways that $a$ becomes unattainable are demonstrated in Figure 6. 


\begin{tabular}{|c|c|c|c|}
\hline Round 1 & Round 2 & Round 1 & Round 2 \\
\hline \multirow[t]{7}{*}{$j \stackrel{a}{\rightarrow} 1$} & $j \stackrel{a}{\rightarrow} 2$ & $1 \stackrel{b}{\rightarrow} j$ & $1 \stackrel{b}{\rightarrow} 2$ \\
\hline & $1 \stackrel{a}{\rightarrow} 3$ & & $j^{a} \stackrel{b}{\rightarrow} 3$ \\
\hline & & $j^{a} \stackrel{a}{\rightarrow} 4$ & $4 \stackrel{a}{\rightarrow} 5$ \\
\hline & & $2 \stackrel{c}{\rightarrow} 6$ & $2 \stackrel{c}{\rightarrow} 7$ \\
\hline & & & $6 \stackrel{c}{\rightarrow} 8$ \\
\hline & & $3 \stackrel{d}{\rightarrow} 9$ & $3 \stackrel{d}{\rightarrow} 10$ \\
\hline & & & $9 \stackrel{d}{\rightarrow} 11$ \\
\hline
\end{tabular}

Figure 6: Let $j^{a}$ be the owner of $a$. On the left, $a$ may be unattainable because in $x^{Q}$ under $E^{\prime}$, three agents are assigned object $a$. On the right, since three agents are assigned $\Omega_{2}=c, 2$ must transfer in R1 and R2; similarly so for 3. This implies $j$ must "help" 1 transfer $b$ to 3 in R2; which further implies $j$ may not transfer $a$ in R2. Hence, only two agents receive $a$ in $x^{Q}$ under $E^{\prime}$, and $a$ is unattainable.

This means there is $u \in U^{0}$, and $u_{z} \in u$ such that at Step $Q$ under $\left.E^{\prime} 1\right) u_{z}$ is the earliest-priority remaining agent, and 2) a points to $u_{z}{ }^{23}$ Furthermore, by definition of $u \in U^{0}$, there is $u_{z-1}, \ldots, u_{1} \in u$ such that at Step $Q$ under $E^{\prime}, u_{z}$ points to $u_{z-2}$ points to... $u_{1}$, and $u_{1} \in J^{0}$. Hence, at Step $Q$ under $E$, a points to $u_{z}$ points to $u_{z-1}$ points to... $u_{1}$ points to $\Omega_{i}$. At Step $Q+1$ under $E$, each of these objects/agents still points in the same fashion. Since at Step $Q+1$ under $E, i$ points to $a, i$ is in a cycle. This contradicts the fact that $V>V^{\prime}=Q+1$.

Case 2: $a=\varphi_{i}\left(E^{\prime}\right)$. At Step $Q+1$ under $E^{\prime}$, the fact that $\Omega_{i}^{\prime}=\emptyset$ and $i$ is in a cycle implies that $i$ is the earliest-priority remaining agent. That is, $i$ is earliest in the set $N^{Q} \backslash\left\{\bigcup_{s \in U^{0} \cup T^{0}} s\right\}$. This implies, at Step $Q+1$ under $E$, either $i$ or an agent in $\bigcup_{s \in U^{0}} s$ is the earliest-priority remaining agent.

Subcase 2.1: At Step $Q+1$ under $E$, an agent in $\bigcup_{s \in U^{0}} s$ is the earliest-priority remaining agent. Let $u_{z} \in u$ be this agent.

Subcase 2.1.1: At Step $Q+1$ under $E, j^{a}$ is not a remaining agent. Then at Step $Q+1$ under $E, a$ points to $u_{z}$. By the reasoning of Case $1, i$ is in a cycle at Step $Q+1$ under $E$.

Subcase 2.1.2: At Step $Q+1$ under $E, j^{a}$ is a remaining agent. At Step $Q$ under $E^{\prime}$, if $j^{a}$ points to an agent in $\bigcup_{s \in U^{0}} s$, then there is $u \in U^{0}$, and $u_{y}, \ldots, u_{1} \in u$ such that $a$ points to $j^{a}$ points to $u_{y}$ points to $u_{y-1}$ points to... $u_{1}$, and $u_{1} \in J^{0}$. By the reasoning of Case $1, i$ is in a cycle at Step $Q+1$ under $E$. At Step $Q$ under $E^{\prime}$, if $j^{a}$ points to either an agent in $\bigcup_{s \in T^{0}} s$ or $\varphi_{j^{a}}\left(E^{\prime}\right)$, then let $b$ be the object $j^{a}$ points to at Step $Q+1$ under $E$.

Subcase 2.1.2.1: $b \neq \varphi_{j^{a}}\left(E^{\prime}\right)$. Since $b$ is unattainable, by the reasoning in Case 1 , at Step $Q$ under $E, b$ points to $u_{z}$. Furthermore, there is $u \in U^{0}$ and $u_{z-1}, \ldots, u_{1} \in u$ such that at Step $Q+1$ under $E$, $a$ points to $j^{a}$ points to $b$ points to $u_{z}$ points to $u_{z-1}$ points to... $u_{1}$ points to $\Omega_{i}$ points to $i$ points to $a$. Then, $i$ is in a cycle at Step $Q+1$ under $E$.

\footnotetext{
${ }^{23}$ Although $a$ points to $u_{z}$, it is not necessarily the case that $a$ is in a cycle at Step $Q$ under $E^{\prime}$. For example, consider the latter situation in Figure 6. Let $x^{Q-1}$ under $E^{\prime}$ be such that each agent each agent except 11 in that transfer process is assigned his respective object. Then, $a$ and $d$ are still attainable given $x^{Q-1}$ under $E^{\prime}$. At Step $Q$ under $E^{\prime}$, if $d$ is in a cycle, then as the transfer process shows, at Step $Q+1$ under $E^{\prime}, a$ is not attainable.
} 
Subcase 2.1.2.2: $b=\varphi_{j^{a}}\left(E^{\prime}\right)$. At Step $Q+1$ under $E^{\prime}$, since $i$ is the earliest-priority remaining agent, $b$ either points to $i$ or an agent $j^{b} \in c\left(i, E^{\prime}\right)$ where $\Omega_{j^{b}}=b$. If $b$ points to $i$, then by definition of Case 2.1, at Step $Q+1$ under $E, b$ points to $u_{z}$ and the reasoning of Case 1 holds. If $b$ points to $j^{b}$, then we consider what $j^{b}$ points to at Step $Q$ under $E^{\prime}$ and follow the reasoning of Subcase 2.1.2, replacing $j^{a}$ with $j^{b}$. Since $c\left(i, E^{\prime}\right)$ is finite, there is eventually an agent $j$ which points to $i$, and we are done.

Subcase 2.2: At Step $Q+1$ under $E, i$ is the earliest-priority remaining agent. Replacing $u_{z}$ with $i$, the reasoning of Case 2.1 holds.

The proof for the general case involves a straightforward generalization of the proof for the case where $V^{\prime}=Q+1$. Now instead of just $U^{0}$ causing unattainability of $a$, a sequence of sets of cycles $U^{0}, U^{1}, \ldots, U^{k}$ causes the unattainability of $a$.

Step 2: $\varphi_{i}(E) R_{i} \varphi_{i}\left(E^{\prime}\right)$. If $V \leq V^{\prime}$, then by Step 1, we are done. Let $V>V^{\prime}$. Let $a$ be the object $i$ points to at Step $V^{\prime}$ under $E$.

Case 1: $a \neq \varphi_{i}\left(E^{\prime}\right)$.

Subcase 1.1: At Step $V^{\prime}$ under $E, j^{a}$ is not a remaining agent.

By Lemma 1 and Step 1, the set of attainable objects at Step $V^{\prime}$ under $E$ contains the set of attainable objects at Step $V^{\prime}$ under $E^{\prime}$. Since $U^{0}, \ldots, U^{V^{\prime}-Q-1}$ are the only extra cycles in $x^{V^{\prime}-Q-1}$ under $E^{\prime}$ compared to $x^{V^{\prime}-Q-1}$ under $E$, these cycles must have "caused" the unattainability of $a$ at some step under $E^{\prime}$. That is, there is a $g \in \mathbb{N}$ such that at Step $Q+g$ under $E^{\prime}, a$ is attainable, but at Step $Q+g+1$ under $E^{\prime}, a$ is not attainable. Let $h \in U^{g}$ be the earliest-priority remaining agent at Step $g$ under $E^{\prime}$; equivalently, $h$ is the earliest-priority remaining agent in the set $N^{Q} \backslash\left\{\bigcup_{\left.s \in T^{0}, \ldots, T^{g-1}, U^{0}, \ldots, U^{g-1}\right\}} s\right\}$. This implies that either $h$ or an agent in $\bigcup_{s \in U^{0}, \ldots, U^{g-1}} s$ is the earliest-priority remaining agent in the set $N^{Q} \backslash\left\{\bigcup_{s \in T^{0}, \ldots, T^{g-1}} s\right\}$ - which is the set of remaining agents at Step $Q+g$ under $E$. Let $h_{z}$ be this agent. By definition, only the cycles $S^{0}, \ldots, S^{V^{\prime}-1}$ occur at Steps $Q, \ldots, V^{\prime}-1$ under $E$; hence, $h_{z}$ is the earliest-priority remaining agent at Steps $Q+g, \ldots, V^{\prime}$ under $E$. Let $\bar{g} \in\{0, \ldots, g\}$ be such that Step $Q+\bar{g}$ under $E$ is the first Step under $E$ at which $h_{z}$ is the earliest-priority remaining agent.

For each $k \in\{0, \ldots, \bar{g}-1\}$, at Step $Q+k$ under $E$, if the earliest-priority remaining agent $j$ is in the set $\bigcup_{s \in U^{0}, \ldots, U^{g}} s$, then $j$ is the earliest-priority remaining agent at Steps $Q+k, \ldots, V^{\prime}$ under $E$. Since we have established $h_{z} \neq j$ is the earliest-priority remaining agent at Step $V^{\prime}$ under $E$, this is not possible. Hence, for each $k \in\{0, \ldots, \bar{g}-1\}$, the earliest-priority remaining agent at Step $Q+k$ under $E$ is in the set $\bigcup_{s \in T^{0}, \ldots, T^{\bar{g}-1}} s$. This implies for each $k \in\{0, \ldots, \bar{g}-1\}$, at Steps $Q+k$ under $E$ and $E^{\prime}$, the earliest-priority remaining agent is the same.

Next, we construct a sequence of agents $h_{z-1}, \ldots, h_{1}$. For Steps $Q, \ldots, Q+g$ under $E^{\prime}$, $h_{z}$ points to a sequence of agents (Figure 7). 


\begin{tabular}{c|ccc} 
Step under $E^{\prime}$ & & & \\
\hline$Q$ & $T^{0}$ & $U^{g_{z}}$ & $U^{0}$ \\
$Q+1$ & $T^{2}$ & $U^{g_{z}}$ & $T^{1}$ \\
$Q+2$ & $T^{2}$ & $U^{g_{z}}$ & $T^{2}$ \\
$Q+3$ & $U^{3}$ & $U^{g_{z}}$ & $T^{3}$ \\
$\vdots$ & $\vdots$ & $\vdots$ & $\vdots$ \\
$Q+g_{z}$ & $U^{g_{z}}$ & $U^{g_{z}}$ & $U^{g_{z}}$
\end{tabular}

Figure 7: Several possible sequences of agents at which $h_{z}$ points to are shown. In the first column, at Step $Q$ under $E^{\prime}, h_{z}$ points to an agent in $\bigcup_{s \in T^{0}} s$. The first Step under $E^{\prime}$ at which $h_{z}$ points to an agent in a " $U$ " cycle is Step $Q+3$. Hence, if the conditions 1.a and 1.b hold, then $h_{z-1}$ is this agent, and $g_{z-1}=3$.

Let $\hat{g} \in\{0, \ldots, g\}$ be such that Step $Q+\hat{g}$ under $E^{\prime}$ is the first Step under $E^{\prime}$ that either 1. for each Step $Q, \ldots, Q+\hat{g}-1$ under $E^{\prime}$,

(a) $h_{z}$ points to an agent in $\bigcup_{s \in T^{0}, \ldots, T^{\hat{g}-1}} s$,

(b) $h_{z}$ does not point to an object in $\bigcup_{s \in U^{0}, \ldots, U^{\hat{g}-1}} \Omega_{s}$, and

at Step $Q+\hat{g}$ under $E^{\prime}, h_{z}$ points to an agent in $\bigcup_{s \in U^{\hat{g}}} s$, OR

2. for each Step $Q, \ldots, Q+\hat{g}$ under $E^{\prime}$,

(a) $h_{z}$ points to an agent in $\bigcup_{s \in T^{0}, \ldots, T^{\hat{g}}} s$, and

(b) at Step $Q+\hat{g}$ under $E^{\prime}, h_{z}$ points to an object in $\bigcup_{s \in U^{0}, \ldots, U \hat{g}-1} \Omega_{s}$.

Such a $\hat{g}$ exists; let $g_{z-1}=\hat{g}$. If $\hat{g}$ satisfies the former condition, then let $h_{z-1} \in \bigcup_{s \in U^{\hat{g}}} s$ be the agent that $h_{z}$ points to at Step $\hat{g}$ under $E^{\prime}$. If the $\hat{g}$ satisfies the latter condition, then let $h_{z-1} \in \bigcup_{s \in U^{0}, \ldots, U^{\hat{g}}} s$ be the owner of the object $h_{z}$ points to at Step $Q+\hat{g}$ under $E^{\prime}$.

By construction, at Step $Q+g_{z-1}$ under $E, h_{z}$ points to $h_{z-1}$. Repeat this process for $h_{z-1}$ to find $h_{z-2}$, and so forth, until we arrive at the first $h_{z-x}$ who is in $\bigcup_{s \in U^{0}} s$.

We show no agent appears twice in this sequence. Suppose by contradiction, there is a consecutive subsequence $h_{k}, h_{k-1}, \ldots, h_{k-x}$ such that $h_{k}=h_{k-x}$. This is only possible if each $h_{k}, \ldots, h_{k-x}$ iteratively satisfies the first condition, implying $g_{k}=g_{k-1}=\cdots=g_{k-x}=g_{k}$. Then, at Step $Q+g_{k}$ under $E, h_{k}, \ldots, h_{k-x}$ forms a cycle. Since each of these agents is not in $\bigcup_{s \in T^{g_{k}}} s$, this is a contradiction. By finiteness of the number of agents, we arrive at $h_{z-x}$ who is in $\bigcup_{s \in U^{0}} s$.

Consider $h_{z-x}$. By definition of $U^{0}, h_{z-x}$ points to an agent in $\bigcup_{s \in U^{0}} s$. Let $h_{z-x-1}$ be this agent. If $h_{z-x-1} \in J^{0}$, then we have reached the end of the sequence. Redefine $z$ and relabel agents so that $h_{z-x-1}=h_{1}$. If $h_{z-x-1} \notin J^{0}$, then repeat this process. By the definition of $U^{0}$ and finiteness of cycles, we eventually reach an agent $h \in J^{0}$. Redefine $z$ and relabel agents so that this agent is $h_{1}$.

By construction, for each $k \in\{0, \ldots, z\}$, at Step $Q+g_{k}$ under $E, h_{k+1}$ points to $h_{k}$. Hence, we have a sequence of agents $h_{z}, \ldots, h_{1}$ such that at Step $g_{z-1}$ under $E, h_{z}$ points to $h_{z-1}$ points to... $h_{1}$ (Figure 8 ). Since $h_{z}$ is the earliest-priority remaining agent at Step $V^{\prime}$ 
under $E$ and $j^{a}$ is not remaining, $a$ points to $h_{z}$ points to $h_{z-1}$ points to... $h_{1}$ points to $\Omega_{i}$ points to $i$ points to $a$, and $i$ is in a cycle. This contradicts the assumption that $a \neq \varphi_{i}\left(E^{\prime}\right)$. We have completed Subcase 1.1.

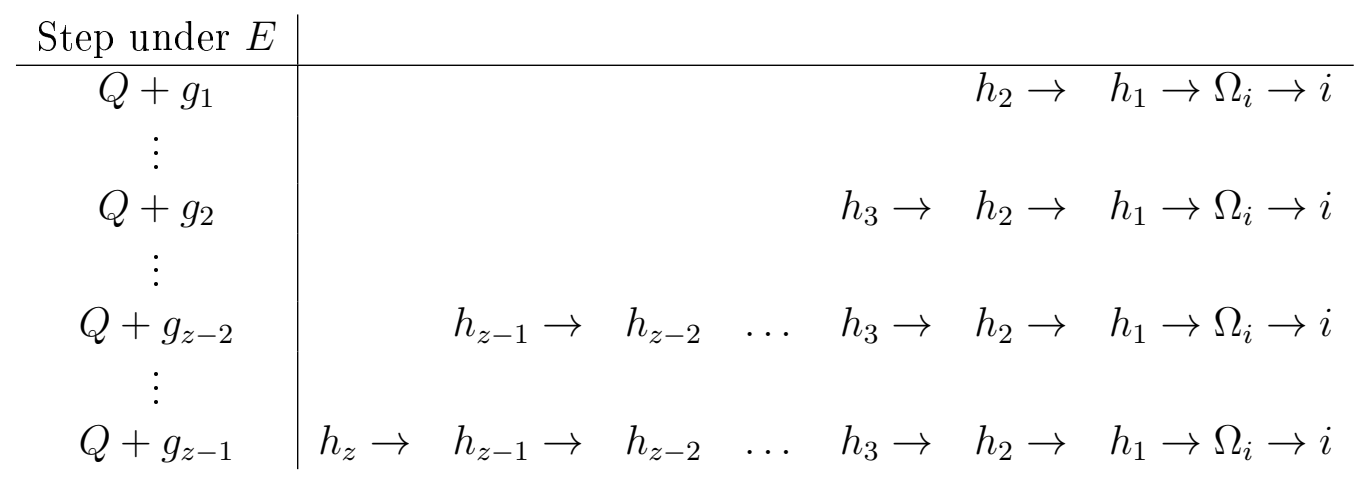

Figure 8: By construction, at each Step in $E$ before Step $Q+g_{z-1}, h_{z}$ only points to agents in who are in cycles $\left(\bigcup_{s \in T^{0}, \ldots, T^{g_{z-1}-1}} s\right)$. So Step $Q+g_{z-1}$ under $E$ is the first Step at which he points to an agent, $h_{z-1}$, not in a cycle. We repeat this argument for $h_{z-1}, \ldots, h_{1}$ until we find $h_{1}$ points to $\Omega_{i}$. Hence, at Step $Q+g_{z-1}$ under $E, h_{z}$ points to $h_{z-1}$ points to... $h_{1}$. The agents still point in this fashion until Step $V^{\prime}$ under $E$.

Subcase 1.2: At Step $V^{\prime}$ under $E, j^{a}$ is a remaining agent. Then, at Step $V^{\prime}$ under $E^{\prime}, j^{a}$ is in a cycle with $i$. Let $h_{z}=j^{a}$. Then, construct $h_{z-1}, \ldots, h_{1}$ as in Subcase 1.1 (the sequence obtained may be different). The reasoning of Subcase 1.1 holds, implying at Step $V^{\prime}$ under $E$, a points to $j^{a}$ points to $h_{z-1}$ points to... $h_{1}$ points to $\Omega_{i}$ points to $i$.

The proof for Step 2, and thereby withholding-proofness, is complete.

\section{Appendix 2}

In sections 2.1-2.4, we show that the properties are independent. In section 2.5, we define a rule outside of the $T T C-S I^{\sigma}$ family satisfying the four properties.

\section{1}

For each $E \in \mathcal{E}$, and each $i \in N$, let $\varphi_{i}(E)=\emptyset$. Then, $\varphi$ satisfies group strategy-proofness, reciprocity lower bound, and withholding-proofness, but not efficiency.

\section{2}

Let $\overline{\mathcal{R}}^{i} \subsetneq \mathcal{R}^{i}$, and $\sigma \in \Sigma$. Let $\pi$ be a permutation of $N$ s.t. $\pi(1)=i$, for each $k \in$ $\left\{2, \ldots, \sigma^{-1}(i)\right\}, \pi(k)=\sigma(k-1)$, and for each $k \in\left\{\sigma^{-1}(i)+1, \ldots, n\right\}, \pi(k)=\sigma(k)$. Let $\pi^{\prime}$ be a permutation of $N$ s.t. $\pi(n)=i$, for each $k \in\left\{1, \ldots, \sigma^{-1}(i)\right\}, \pi(k)=\sigma(k)$, and for each $k \in\left\{\sigma^{-1}(i)+1, \ldots, n\right\}, \pi(k)=\sigma(k-1)$.

For each $E=(R, \Omega) \in \mathcal{E}$, and each $\sigma \in \Sigma$, let 


$$
\varphi^{\sigma}(E) \equiv \begin{cases}T T C-S I^{\pi}(E) & \text { if } R_{i} \in \overline{\mathcal{R}}^{i} \\ T T C-S I^{\pi^{\prime}}(E) & \text { if } R_{i} \in \mathcal{R}^{i} \backslash \bar{R}^{i}\end{cases}
$$

Then, $\varphi^{\sigma}$ satisfies withholding-proofness, the reciprocity lower bound, and efficiency, but not group strategy-proofness. It is straightforward to construct an economy $E$ where $i$ has incentive to misreport.

\section{3}

Let $\hat{\mathcal{E}}$ be the set of $E=(R, \hat{\Omega}) \in \mathcal{E}$ s.t.

- $N=\{1, \ldots, 5\}$,

- $\hat{\Omega}=(a, b, c, d, e)$,

- for each $z \in \mathcal{O} \backslash\{e\}$, e $R_{1} z$, and

- for each $i \in N \backslash 1$, and each $z \in \mathcal{O} \backslash\{a\}, a R_{i} z$.

Let $\sigma(i)=i$, and for each $E \in \mathcal{E}$, let $R_{5}(2)$ be 5's second-ranked object w.r.t. $R_{5}$ and

$$
\varphi(E) \equiv \begin{cases}\left(e, a, a, a, R_{5}(2)\right) & \text { if } E \in \hat{\mathcal{E}} \\ T T C-S I^{\sigma}(E) & \text { if } E \in \mathcal{E} \backslash \hat{\mathcal{E}}\end{cases}
$$

Then, for each $E \in \hat{\mathcal{E}}, \varphi_{1}(E)=\Omega_{5}$, and $\Omega_{1}=a P_{5} e=\varphi_{5}(E)$, in violation of the reciprocity lower bound. It is clear that the rule satisfies efficiency and withholding-proofness.

Claim $\varphi$ satisfies group strategy-proofness.

Proof: Suppose by contradiction that there is $E=(R, \Omega) \in \mathcal{E}, S \subseteq N$, and $R_{S}^{\prime} \in \mathcal{R}^{S}$ such that for each $i \in S, \varphi_{i}\left(R_{S}^{\prime}, R_{-S}, \Omega\right) R_{i} \varphi_{i}(E)$, and for some $j \in S, \varphi_{j}\left(R_{S}^{\prime}, R_{-S}, \Omega\right) P_{j} \varphi(E)$. Let $E^{\prime}=\left(R_{S}^{\prime}, R_{-S}, \Omega\right)$.

Case 1: $E=(R, \Omega) \in \hat{\mathcal{E}}$. If $E^{\prime} \in \hat{\mathcal{E}}$, then $\varphi\left(E^{\prime}\right)=\varphi(E)$, so let $E^{\prime} \in \mathcal{E} \backslash \hat{\mathcal{E}}$. For each $i \in N \backslash 5, \varphi_{i}(E)=\arg \max R_{i}$, so 1) for each $i \in S \backslash\{5\}, \varphi_{i}(E)=\varphi_{i}\left(E^{\prime}\right)$, and 2) $5 \in S$ and $\varphi_{5}\left(E^{\prime}\right)=a P_{5} \varphi_{5}(E)$. First, $\{2,3,4\} \subset S$ is not true, otherwise, by assumption $\varphi_{2}\left(E^{\prime}\right)=\varphi_{3}\left(E^{\prime}\right)=\varphi_{4}\left(E^{\prime}\right)=a$, so $\varphi_{5}\left(E^{\prime}\right)=a$ is not possible. Hence, there is $j \in\{2,3,4\}$ such that $j \notin S$. If each $i \in S$ top-ranks $\Omega_{j}$, then there is some agent $h$ in $S$ such that $\varphi_{h}(E) P_{h} \varphi_{h}\left(E^{\prime}\right)=\Omega_{j}$. If not every agent in $S$ top-ranks $\Omega_{j}$, then the unique efficient allocation for $E^{\prime}$ is the one where each agent is assigned his top-ranked object in $\left(R_{S}^{\prime}, R_{-S}\right)$. Since $E^{\prime} \in \mathcal{E} \backslash \hat{\mathcal{E}}$, there is $i \in S$ such that the top-ranked object in $R_{i}$ and $R_{i}^{\prime}$ is not the same. If $i \neq 5$, then this violates $\varphi_{i}(E)=\varphi_{i}\left(E^{\prime}\right)$; if $i=5$, then $i$ does not top-rank $a$, and $\varphi_{5}\left(E^{\prime}\right) \neq a$-violating the assumption that 5 strictly benefits. 
Case 2: $\quad E=(R, \Omega) \in \mathcal{E} \backslash \hat{\mathcal{E}}$. If $E^{\prime} \in \mathcal{E} \backslash \hat{\mathcal{E}}$, then since $T T C-S I^{\sigma}$ is group-strategyproof, existence of such a coalition $S$ is not possible. Let $E^{\prime} \in \hat{\mathcal{E}}$. If there is a unique efficient allocation, then each agent is assigned their top-ranked object and manipulation is impossible. So suppose there are multiple. This implies that there is $i, j \in N$ such that all agents $N \backslash\{i\}$ top-rank $\Omega_{i}, \varphi_{j}(E) \neq \Omega_{i}$, and for each $h \in N \backslash\{i, j\}, \varphi_{h}(E)=\Omega_{i}$. Note that $j$ is assigned his second-ranked object, and each other agent is assigned their top-ranked object. By definition of $S, \varphi_{j}\left(E^{\prime}\right)=\Omega_{i}$. If $i=1$, then since $E \in \mathcal{E} \backslash \hat{\mathcal{E}}, 1$ does not top-rank $e$, implying $1 \in S$, and $\varphi_{i}(E) P_{i} e=\varphi_{i}\left(E^{\prime}\right)$-a contradiction. If $i=5$, then all agents $N \backslash\{5\}$ top-rank $e$ in $R$, and $\varphi_{j}\left(E^{\prime}\right)=e$. Since $j \neq i=5$, and $e \notin \bigcup_{h \in\{2,3,4\}} \varphi_{h}\left(E^{\prime}\right), j=1$. Then, since 2 top-ranks $e$ in $R$, but from $E^{\prime} \in \mathcal{E} \backslash \hat{\mathcal{E}}, 2$ top-ranks $a$ in $R^{\prime}$, so $2 \in S$ and $\varphi_{2}(E)=e P_{i} a=\varphi_{2}\left(E^{\prime}\right)$ - a contradiction. If $i \in\{2,3,4\}$, then all agents $N \backslash\{i\}$ top-rank $\Omega_{i}$ in $R$. Since $\Omega_{i} \notin \bigcup_{h \in\{1,2,3,4\}} \varphi_{h}\left(E^{\prime}\right), \varphi_{j}\left(E^{\prime}\right)=\Omega_{i}$ implies $j=5$. Hence, $1 \in S$, and $\varphi_{1}(E)=\Omega_{i} P_{1} e=\varphi_{1}\left(E^{\prime}\right)-$ a contradiction.

\section{4}

Let $\sigma \in \Sigma$. Let $\pi$ be a permutation of $N$ s.t. $\pi(1)=i$, for each $k \in\left\{2, \ldots, \sigma^{-1}(i)\right\}, \pi(k)=$ $\sigma(k-1)$, and for each $k \in\left\{\sigma^{-1}(i)+1, \ldots, n\right\}, \pi(k)=\sigma(k)$. Let $\pi^{\prime}$ be a permutation of $N$ s.t. $\pi(n)=i$, for each $k \in\left\{1, \ldots, \sigma^{-1}(i)\right\}, \pi(k)=\sigma(k)$, and for each $k \in\left\{\sigma^{-1}(i), \ldots, n-1\right\}$, $\pi(k)=\sigma(k+1)$.

For each $E=(R, \Omega) \in \mathcal{E}$, and each $\sigma \in \Sigma$, let

$$
\varphi^{\sigma}(E) \equiv \begin{cases}T T C-S I^{\pi}(E) & \text { if } \Omega_{i} \neq \emptyset \\ T T C-S I^{\pi^{\prime}}(E) & \text { if } \Omega_{i}=\emptyset\end{cases}
$$

Then, $\varphi^{\sigma}$ satisfies group strategy-proofness, the reciprocity lower bound, and efficiency, but not withholding-proofness.

\section{5}

Let $\sigma, \sigma^{\prime} \in \Sigma$ be s.t. $\sigma(2)=\sigma^{\prime}(3), \sigma(3)=\sigma^{\prime}(2)$, and for each $i \in N \backslash\{2,3\}, \sigma(i)=\sigma^{\prime}(i)$. Let $\{A, B\}$ be a partition of $\mathcal{O}$.

For each $E \in \mathcal{E}$, let

$$
\varphi(E) \equiv\left\{\begin{array}{ll}
T T C-S I^{\sigma}(E) & \text { if } T T C-S I_{1}^{\sigma}(E) \in A \\
T T C-S I^{\sigma^{\prime}}(E) & \text { if } T T C-S I_{1}^{\sigma}(E) \in B
\end{array} .\right.
$$

Then, $\varphi$ satisfies group strategy-proofness, withholding-proofness, efficiency, and the reciprocity lower bound, but $\varphi^{\sigma}$ is not in the $T T C-S I$ family of rules. 


\section{References}

[1] A. Abdulkadiroğlu and T. Sönmez. House allocation with existing tenants. Journal of Economic Theory 88, 1999 (233-260).

[2] E. Adar and B. A. Huberman. Free riding on gnutella. First Monday 5.10, 2000.

[3] F. Ahmed-Zaid, et al. Vehicle safety communications-applications (VSC-A) Final Report. US Department of Transportation, DOT HS 811 492A, 2011.

[4] H. Anno. A short proof for the characterization of the core in housing markets. Economics Letters 126, 2015 (66-67).

[5] M. Atlamaz and B. Klaus. Manipulation via endowments in exchange markets with indivisible goods. Social Choice and Welfare 28.1, 2007 (1-18).

[6] B. Baker and R. Shostak. Gossips and Telephones. Discrete Mathematics 2, 1972 (191193).

[7] H. Konishi, T. Quint, and J. Wako. On the Shapley-Scarf economy: the case of multiple types of indivisible goods. Journal of Mathematical Economics 35, 2001 (1-15).

[8] M. Feldman and J. Chuang. Overcoming free-riding behavior in peer-to-peer systems. ACM Sigecom Exchanges 6.1, 2005 (41-50).

[9] F. Li and Y. Wang. Routing in vehicular ad hoc networks: a survey. IEEE Vehicular Technology Magazine 2.2, 2007 (12-22).

[10] J. Ma. Strategy-proofness and the strict core in a market with indivisibilities. International Journal of Game Theory 23.1, 1994 (75-83).

[11] S. Pápai S. Strategyproof assignment by hierarchical exchange. Economentrica 68.6, $2000(1403-1433)$.

[12] A. Postlewaite. Manipulation via endowments. Review of Economic Studies 46.2, 1979 (255-262).

[13] M. Pycia and U. Ünver. Incentive compatible allocation and exchange of discrete resources. Working Paper, 2015.

[14] A. Roth. The college admissions problem is not equivalent to the marriage problem. Journal of Economic Theory 2.36, 1985 (277-288).

[15] L. Shapley and H. Scarf. On cores and indivisibility. Journal of Mathematical Economics 1.1, 1974 (23-37).

[16] T. Sönmez. Strategy-proofness and essentially single-valued cores. Econometrica 67.3, 1999 (677-689). 
[17] T. Sönmez and U. Ünver. House allocation with existing tenants: a characterization. Games and Economic Behavior 69.2, 2010 (425-445).

[18] W. Thomson. The equilibrium allocations of Walras and Lindahl manipulation games. Discussion Paper No. 111, Center for Economic Research, Department of Economics, University of Minnesota, 1979.

[19] W. Thomson. Borrowing-proofness of the Lindahl rule in Kolm triangle economies. Working Paper, 2010. 